\title{
Charakteristik des judenspanischen Dialekts von Saloniki.
}

\section{Vorbemerkung.}

Die vorliegende Charakteristik des isp. Dialekts von Saloniki berubt auf Sammlungen, die ich in den Jahren 1916-18 als Soldat im Verkehr mit einer Üsküber, aus Saloniki eingewanderten jsp. Familie gemacht habe.

Das 17 jährige Fräulein Bella Assael war so liebenswürdig, mir die drei aufgezeichneten Märchen zu erzählen. Sie konnte recht gut Französisch (als Schülerin einer in Friedenszeiten von der Alliance Israelite Universelle unterhaltenen französischen Schule), etwas Serbisch und Bulgarisch, sehr wenig Türkisch und nicht ein Wort Hebräisch.

\section{Literaturverzeichnis.}

\section{Romanischer Teil.}

Cuervo R. I., Apuntaciones Críticas sobre el Lenguaje Bogotano. $5^{\text {a }}$ edición. Paris 1907. = Cuervo Ap.

- Diccionario de Construcción y Régimen de la Lengua Castellana. A-D. Paris 86-93. = Cuervo D.

Diccionario de la Lengua Castellana por la Real Academia Espanola XIII ed. Madrid 1899. = Dicc. Ac.

Espinosa A. M., Studies in New Mexican Spanish I u. Il., Revue de Dial. Rom. I (1909), S. 157 f.; IIr (1911), S. 251 ff.

Grünbaum M., Jüdisch-spanische Chrestomathie. Frankfurt a. M. 1896.

Hanssen Fr., Spanische Grammatik auf historischer Grundlage. Halle 1910. $=$ Hanssen.

Lamouche L., Queiques mots sur le dialecte espagnol parlé par les Israélites de Salonique. Rom. Forsch. XXIII (1907), p. 969-991. = Lamouche.

Menéndez-Pidal R., Manual Elemental de Gramática Histórica Española. 22 ed. Madrid 1905. = Men. Man.

- Cantar de Mió Cid, Texto, Gramática y Vocabulario I. II. III. Madrid 1908-11. = Men. Cid.

Meyer-Lübke W., Grammatik der romanischen Sprachen. Leipzig 1890 -99. = M.-L. I-III.

- Romanisches etymologisches Wörterbuch. Heidelberg 1911-20. $=M_{0} \cdot$ L. Wb. 
Subak J., Zum Judenspanischen, ZRPh. XXX (1906), S. 129-185. = Subak Judsp.

- Das Verbum im Judenspanischen. In: Bausteine zur romanischen Philologie. Festgabe für Adolfo Mussafia. Halle 1905, S. 321-33I. = Subak Verbum.

- Judenspanisches aus Salonikki, mit einem Anhange: Judenspanisches aus Ragusa. Triest 1906. = Subak Sal.

- Vorläufiger Bericht über eine im Auftrag der Balkan-Kommission der kais. Akademie der Wissenschaften zu Wien unternommene Forschungsreise nach der Balkanhalbinsel zur schriftichen und phonographischen Aufnahme des Judenspanischen. Anzeiger der philos.-histor. Klasse der kais. Akad. d. Wiss. in Wien, Jg. I910, Nr. VI.

Tobler A., Vermischte Beiträge zur französ. Grammatik I\&, II, III?, IV. Leipzig 1902-08. = Tobler V. B.

Wagner M. L., Beiträge zur Kenntnis des Judenspanischen von Konstantinopel. Schriften der Bilkankommission. Ling. Abtlg. XI. = Wagner.

- Los Judios de Levante. Revue de Dial. Rom. S. 470-506.

Weigert B., Untersuchungen zur span. Syntax auf Grund der Werke des Cervantes. Berlin 1907. = Weigert.

Zauner Ad., Altspan. Elementárbuch. Heidelberg 1908. = Zauner.

\section{Orientalischer Teil.}

Beer G., Hebräische Grammătik I-II, Sammlung Göschen. Berlin-Leipzig 1915 .

Birnbaum S., Praktische Grammatik der jiddischen Sprache für den Selbstunterricht. Wien k. Leipzig 1915.

Dahon Abr., Essai sur les rocables turcs dans le judéo-espagnol. In: Keleti Szemle (Revue Orientale) 1903, S. $215 \mathrm{ff}$. u. 1904, S. I I I ff.

Gesenius-Buhl, Hebräisches u. aramäisches Handwörterbuch über das alte Testament. Leipzig $1915^{16}$.

Gesenius-Ka utzsch, Hebräische Grammatik. Leipzig $1909^{28}$.

Ja cob G., Zur Grammatik des Vulgärtürkischen. In: Zeitschr. d. Deutschen Morgenländischen Gesellschaft, Bd. LII (1898), p. 695-729.

Horten M., Kleine türkische Sprachlehre. Heidelberg 1916.

Levy, Neuhebräisches u. chaldäisches Wörterbuch. Leipzig 1876-89.

Meyer Gustav, Etymol. Wörterbuch der albanesischen Sprache. Strafsburg 189 I. Ružićka-Ostoić C., Türkisch-deutsches Wörterbuch. Wien 1879.

Strack Hermann L., Hebräische Grammatik mit Übungsbuch. München $1917^{18}$.

- Jüdisches Wörterbuch. Mit besonderer Berücksichtigung der gegenwärtig in Polen üblichen Ausdrücke. Leipzig 1916.

Tewfik Hakki, Türkisch-deutsches Wörterbuch. Leipziğ 1907. = Tewfik. (Die arabischen Ziffern hinter dem Namen bezeichnen die Seite, die römischen die Spalte, auf der sich das zitierte Wort befindet.)

Weil Gotthold, Grammatik der Osmanisch - türkischen Sprache. Berlin 1917. Zenker, Türkisch-arabisch-persisches Handwörterbuch. Bd. I. 2. Leipzig 1866-76. = Zenker. 


\section{Erklarung der Abkurzungen und Transkriptionszeichen.}

1. Abkürzungen.

$$
\begin{aligned}
& \text { aport. = altportugiesisch } \\
& \text { asp. = altspanisch } \\
& \text { dtsch. = deutsch } \\
& \text { frz. = französisch } \\
& \text { hebr. = hebräisch } \\
& \text { jsp. = judenspanisch }
\end{aligned}
$$

$$
\begin{aligned}
& \text { lat. = lateinisch } \\
& \text { nhebr. = neuhebräisch } \\
& \text { nsp. = neuspanisch } \\
& \text { port. = portugiesisch } \\
& \text { salon. = salonikisch } \\
& \text { tk. = türkisch. }
\end{aligned}
$$

\section{Transkriptionszeichen.}

Die Transkription entspricht der kroatischen' Schreibweise. Folgende Zeichen kommen hinzu :

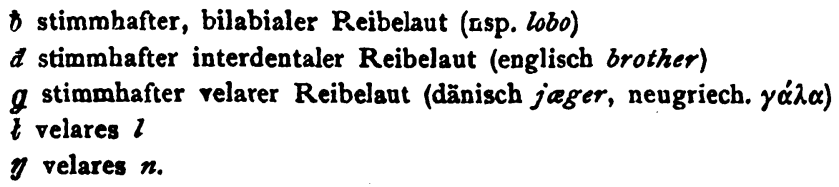

Die Qualität von betontem $e$ und $O$ ist in der üblichen Weise durch $e, \rho$ und $\&, q$ bezeichnet.

\section{Einleitung.}

Jede Arbeit über einen jsp. Dialekt wird in erster Linie von der Frage auszugehen haben, inwieweit das gesammelte sprachliche Material asp. Erbgut darstellt, und dann versuchen müssen, alle Abweichungen vom Altspanischen, sei es als spontane jüdische Neuschöpfungen, sei es als Entlehnungen aus anderen Sprachen, zu erklären.

Das Altspanische und nicht das Lateinische oder Arabische mufs daher die Grundlage einer wissenschaftichen Behandlung des Jsp. bilden, wie Wagner mit Recht bei Besprechung der Subakschen Arbeiten hervorhebt (Reoue de Dial. Rom. I, 494).

$\mathrm{Da}$ Wagner selbst diese methodische Einstellung seinen ${ } B e i-$ trägen sur Kenntnis des Judenspanischen won Konstantinope/" zul Grunde gelegt hat, glaubte die vorliegende Charakteristik des Salonikier Dialekts sich eng an dieses Werk anschliefsen und auf dort Behandeltes nur kurz verweisen zu sollen.

Sie enthält als ersten Teil drei Märchen in phonetischer Notierung mit gegenüberstehender deutscher Übersetzung. Die Grammatik, die den zweiten Teil der Arbeit bildet, macht auf irgend welche Vollständigkeit keinen Anspruch, sondern beschrânkt sich im wesentlichen auf Feststellung und Einordnung der in den Texten anzutreffenden sprachlichen Erscheinungen. Ein Glossar verzeichnet die aus irgend einem Grunde Interesse beanspruchenden Wörter.

1 Mit $h$ wird im Kroatischen der stimmlose vel a re Reibelant (dtsch. ach, nsp. ojo), nicht der laryngale bezeichnet.

Zeituchr. f. rom. Phil. XL. 


\title{
Erster Teil : Drei judenspanische Murchen.
}

\author{
I. \\ El asniko del hahdn.
}

Gran bugnoz 1 de un hahän. Akgl hahán tenia un asniko. Un dia de sabd el se hye al kal $i$ en su kaza abia kriaturas ke estaban ğugando kon el azniko. En ğugando el bpto del asno' se kaiag 3 al ppğo.

Kuando el hahdn atornp del kal, las kriaturas kurizigron en de gl $i$ le dišseron: 'Siñor hahan, un asniko kaìg al pgğo, se pug̨e kitarlo

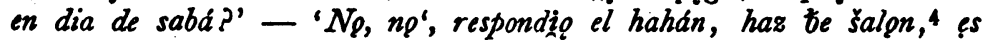
pekado grande, fuięndobos de akt.

El uno's de las kriaturas le diš: 'Siñor hahdn, mire ke el azniko 10 es suio!' - 'Cū̄igendo pręsto, kitándolo, antes ke se afoge.

\section{II.}

\section{El ręi $i$ el tezir.}

El ręi $i$ el bezir estaban etando moabęl. ${ }^{6}$ El ręi dizía ke las kriaturas ia nasen sabidas. El bezir al kontrarizo dizia ke las kriaturas lo ke siçnten ambzzan.

Un dia la reina estaba para parir $i$ pario un fizo. El ręi le 5 dìg a una kriadẹra i le akabide ke no fable nada delantre el nino. La kriadera fiso lo ke el regi le epkomendp: No le dizia, no le kantata, no le ğugata nada.

En frẹte del konák del rę̧ ęra la mar. Un dia un gudtíp probe pase delantre las tentanas de pnde estata el nitito. El dezesperado ombre diso entre si: "If to a "čar la fesesika mia tres tęsez en allo. I si ez mazdl ke ne me ęte a la mar, la to a aferar todas trẹz tesez. I ansina fue. En kada bes ke exaba la fes, disia 'Qpala!'

1 Eran buenos ..., der gewöhnliche Anfang der salon. Märchen. Um zum Verständnis der Redensart zu gelangen, wird man von Sätzen wie neran buenos de dos ermanos" ausgehen mǘssen und darin ein de seben, das nlogisches Subjekt einfübrt" (Tobler, V. B. I, 5 ff.), also: "Es waren Gute, von swei Brïdern her". Doch habe ich für diese Verwendung von de keine asp. Beispiele. Der Sinn der Konstruktion scheint sich früh verdunkelt zu haben. ${ }_{n}$ Es waren einmal 2 wei gute Brüder" beifst heute salon. eran buenos de dos buenos ermanos. Ein zu eran buenos gesetzter logisch nicht zu rechtfertigender Singular (wie im Text) hat den Ûsküber Dialekt anscheinend $2 n$ der allerdings anch nicht analysierbaren Form era bueno de ... gefübrt, wo man wenigstens $u n$ bueno erwarten würde, und dieser dann auch bei pluralischem logischem Subjekte Geltung verschafft.

2 el boto del asno. Wie la coquine de Toinette. Tobler, V. B. I, 135, Hanssen $\S 43,5$, Wagner $\S 67$. 
I.

Das Eselchen des Rabbis.

Es war einmal ein Rabbi. Dieser Rabbi hatte ein Eselchen. An einem Sabbath-Tage ging er zur Synagoge, und bei ihm zu Hause waren Kinder, die mit dem Eselchen spielten. Wie sie spielten; fiel der dumme Esel in den Brunnen.

Als der Rabbi von der Synagoge zurückkam, liefen die Kinder zu ihm und sprachen: „Herr Rabbi, ein Eselchen ist in den Brunnen gefallen, darf man es am Sabbath herausholen?" - "Nein, nein", antwortete der Rabbi, "Gott behüte, das wäre eine grofse Sünde, macht euch fort!

Da sprach eins von den Kindern: "Herr Rabbi, so seht doch, es ist ja Euer Eselchen!". "So lauft schnell und holt es heraus, ehe es ertrinkt!".

II.

Der König und der Wesir.

Der König und der Wesir unterhielten sich. Der König sagte, dars die Kinder bereits gebildet auf die Welt kommen. Der Wesir dagegen behauptete, dafs die Kinder lernen, was sie [sprechen] hören.

Eines Tages sollte die Königin niederkommen, und sie gebar einen Sohn. Der König übergab ihn einer Amme und verbot ihr, etwas in Gegenwart des Kindes zu sprechen. Die Amme tat, wie ihr der König befohlen hatte. Sie sagte ihm nichts, sang ihm nichts und spielte nichts mit ihm.

Vor dem Palaste des Königs war das Meer. Eines Tages ging ein armer Jude vor den Fenstern [des Palastes] vorbei, an denen sich das Kind befand. Der arme Verzweifelte sprach bei sich: "Ich will mein Feslein dreimal hochwerfen. Und wenn es mir nicht bestimmt ist, dars ich mich ins Meer stürzen soll, werde ich es alle drei Male wieder auffangen." Und so geschah es. Jedesmal wenn er den Fes hochwarf, rief er ${ }_{\text {HHoppla!" }}$ Wesen.

s se kaip, Zeile 6 kail. Das Reflexirum bei Personen oder personifizierten

4 haz te salgn. On „Fernhaltung" (nhebr.) ve " "und", salon [für salom,

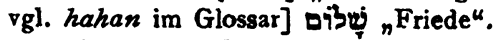

s el uno. Das Maskulinum durch die Vorstellung hervorgervfen, dafs ein Junge zu dem Rabbi spricht.

- ećando moabet: Die Verwendung von ečar an Stelle von fazer (= tk. إنعلك etmek) dürfte sich durch Analogie von ećar gritos, eciar suspiros erklären. Auch die Redensarten ećar lagrimas und ecar ocadas erinnern in ihrer Bildungsweise an die im Türkischen so beliebte Verb-Schöpfung aus Substantiv + etmek. Eine abgeschwächte, dem Begriff jazer naise kommende Bedeutung zeigt ečar auch in Verbindung mit folgenden Substantiven, die ich dem Dicc. Ac. s. v. echar entnommen habe: [echar] olor, sangre, chispa, raices, hojas, flores, frutos, dientes, pelo, bigote, calculo, cuenta, comedia, maldiciones, versos, bravatas, juramentos. 
En laz tentanas ar̃ita estata el fižo del rę̇. Apenas bido lo ke el ğudì? estata fasigndo, kiž fazer lo mizmo, se kitg el tapęzo de

15 su katessa $i$ la etg en atto kemo abia bisto, grilando 'Qpala'. Ma ne aferg de mugto su karapusa [harati] ke kaíg abašo.7 El ğudịg en biendo la karapusa le paresiag ke es el Dip ke le embio parás pqrke la karapusa estata ieqna de dukados. El se hug bolado de la gustada. 8

La kriatura estaba sięmpre repetando ${ }^{9}$ la mizma palabra 'opala'. El ręi marabiżado demande a la kriadęra si es ęia ke le amberg esta palabra. Ma ęia dišo ke nada.

En este punto bi̊do el rej ke el capęo no estata en su katẹsa. Eč̣ un pregen en la sivdá entẹra, kį̣n es ke topí el capęo de la

25 kriatura.

El ğud̈is probe ke abia topado la karapusa, kedld pensatible $i$ diso entre si: 'Kale sẹa ke esta karapusa no es el Dip ke me la 'mbiap. Por siguro ke la karapusa es del fižo del ręi.

lo krego ke en ìetdndola b'a tener un buen regalo, $i$ la jejo.

El ręi le demandg a el ombre donde le bino esta karapusa. El ğudig le konto su estorial.

Ansina el resi entendig ke a las kriaturas kale ambezado 10 a

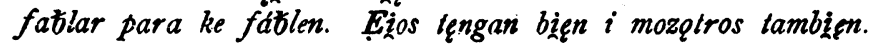

III.

El ręi i las tress ermanas. 1

Eran bugnos de un reji. E़ste rei eč un día un preggn en la sivda" ke ningunos no asiegndan lus 12 esta note. En esta sivda abia tres ermanas probes $i$ laboraderas. Estas no pudian comer el dia si no latoratan la npte. Ansina kalta ke șias laboraban ${ }^{13}$ tambien essia

5 nę̆e para komer el día.

La mas cika d'ejas ke era mui bita, mui zerka i muj inteližgnte diso: 'tamos a meter ait el gasesiko $i$ moz tamos a arodedr tedas tres, $i$ ansina te esle sigura ke ningunos no mez tan a tegr'.

- en tiendo le paresip: in der gesprochenen Sprache nicht selten anzutreffendes Anakoluth, auch franz. vgl. z. B. Strohmeyer, Franz. Schulgrammatik $1919^{2}, \S 221$ b.

- bolado de la gustada: Ausgangspunkt bildet das sebr bäufige bolado de alegrta [also ngeflogen vor Freude ${ }^{\mu}$ ], das in Verbindung mit ser und Verben der Bewegung asp. möglich war (vgl. M.-L. III, \$294 u. \$\$ 308/9) und leicht

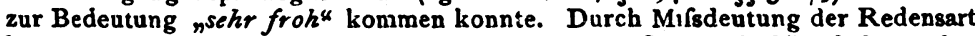
hat salon. anscheinend auch isoliertes bolado den Sinn ${ }_{n}$ froh $h^{\mu}$ erhalten, den de la gustađa (über den Genufs) erläuternd verstärkt.

- repetando . repetar Gallizismus.

10 kale ambesado. Salon. bäufige Konstruktion. Ebenso: se kiere (oder kale) arogado para tomar una koza de el (man muls ihn bitten, um etwas von ihm zu bekommen) oder: Kale kada dia ido a kaminar para estar saludpro. [man mufs jeden Tag spazieren geben, um gesund zu sein]. Ähnlich M.-L. III, 
An den Fenstern oben war der Königssohn. Kaum hatte er gesehen, was der Jude tat, als er es ebenso machen wollte: Er nahm seinen Hut vom Kopfe, warf ihn hoch, wie er's gesehen hatte, und rief dabei: "Hoppla!" Er fing aber sein Hütlein nicht wieder auf, und es fiel herunter. Als der Jude das Hütchen sah, da dünkte es ihm, dafs Gott ihm Geld schickte, denn das Hütlein war voller Dukaten. Voller Freude ging er davon.

Das Kind wiederholte danernd das gleiche Wort: "Hoppla!" Verwundert fragte der König die Amme, ob sie ihm das Wort beigebracht habe. Aber sie sagte nein.

In diesem Augenblick sah der König, dafs [das Kind] keinen Hut mehr auf hatte. Er liefs in der ganzen Stadt ausrufen, wer den Hut des Kindes gefunden hätte.

Der arme Jude, der das Hütlein gefunden hatte, wurde nachdenklich und sprach bei sich: "So muls mir doch nicht Gott das Hütlein geschickt haben! Sicher ist es das Hütlein des Königssohns.

Ich glaube, wenn ich es hinbringe, werde ich eine gute Belohnung erhalten, und er brachte es hin.

Der König fragte den Mann, wie er zu dem Hütlein käme. Der Jude erzählte ihm seine Geschichte.

So begriff der König, dafs man die Kinder sprechen lehren mufs, damit sie sprechen. Thnen möge es gut gehen und uns auch!

\section{III.}

\section{Der König und die drei Schwestern.}

Es war einmal ein König. Dieser König liefs eines Tages in der Stadt ausrufen, dafs in der folgenden Nacht niemand Licht anzünden sollte. In dieser Stadt lebten drei arme, fleifsige Schwestern. Diese hatten am Tage nicht zu essen, wenn sie nicht [auch] Nachts arbeiteten. So mulsten sie auch in dieser Nacht arbeiten, um am Tage essen [zu können].

Die jüngste von ihnen, die sehr lebhaft, sehr verschlagen und sehr klug war, sprach: "Wir wollen hierher das Lämpchen stellen und uns alle drei herumsetzen, und so bin ich ganz gewirs, dafs niemand uns sehen wird."

$\oint 311$ sizilian. vuliri $+t$-Partizipium. Die Konstruktion findet sich bereits im Volkslatein (Raffael Kühner, Lat. Gram.2 [1912], § 127, 11, Anm. 4) und ist auch dem Deutschen nicht fremd. (J. Grimm, Dtsche Gramm. IV. Neuer Abdr. 1898, S. 150f.). Asp. Beispiele habe ich nicht. - Das Tempus von kale hier grammatisch richtig, da es sich um eine ,allgemein gültige Wahrheit" handelt. (Vgl. $§ 35$ u. Hanssen, S. 102).

11 Das Märchen zeigt starke Verwandtschaft mit dem Märchen von den "Neidischen Schwestern" aus "Tausendundeine Nacht ". Uber dessen Verbreitung vgl. Chauvin, Bibliographie des ouvrages relatifs aux Arabes VII, $95 \mathrm{ff}$.

12 lus, ohne Artikel, da asp. ${ }_{n}$ das Accusativ-Objekt erst allmählich den Artikel bekommt". (Men. Cid. I, S. 300).

1s latoratan. Indikativ sehr auffallig. 
Essla note el fizo del ręz salíp el mizmo a espionar la sivdá, $i$ 10 pase guusto delantre de la kasa de las tręz ermanas. Be ke oi lus $i$ se metīg a bęr por el buīako de la puerla i sintīg ke las trẹz ermanas estaban fablando.

Elias por ne siklearse la nęce tomatan a fablar. La una de las tress, la mas grande eto un suspiro $i$ dišs: ' $A$, si el fižo del rgi me

15 tomaba a mi por muzęr, ig le ia a fazer a ęl una kanta mǔ̌o grande ke todo el mundo se ečara $i$ inda ke uḃęra lugar'.

La segunda diš: 'Si a mi el fižo del ręi me tomata, ì le fasia an 14 el una kętía muča grande, ke tọdo el mundo se kutižara i inda ke utiegra lugar'.

20 La mas čika dišo: 'I $\mathrm{n}$ n! Ig le ia a parir an $\mathrm{el}$ dos kriaturas ke tengan en el megio una estrẹia $i$ una luna'.15

TQdo esto el fizo del rei estaza sintįendo. Se fue a su kaza, i a la mañana embio a iamar a las trẹs ermanas.

An eias les paresio ke el rei las embio a iamar porke asindieron

25 lus. Estaban mui espantadas. No kierian ir, ma hueron mui forsadas i hueron. ${ }^{16}$

El ręi lez demando ke koza estutigieron fablando la noče. Eijas se estatan atruensando de fatlar. El rę̣i las menasp i ejas fatlaron.

La grande diso: ' $S i$ tu me tomas a mi, iq te ta a fasegr una 30 kama grande ke tod̄o el mundo se ě̃ara i inda ke ubigra lugar'.

La segunda diso: 'Io disi ke si tu me tomatas, io te ta a fazer una kgť́a mui grande ke el mundo intẹro se kutizara $i$ inda ke ubil ra lugar'.

I la tika diso: 'Si th me lomatas a $\mathrm{mi}$, iq te ia a parir dos 35 kriaturas ke tutigron en el mepio una 'stręia i una luna'.

El rę las kižo a proßar, si ęias dỉseron la berda i dišo a la tika: 'If te t'a tomar a ti por mutér para si tu ablas la terda'.

1 ansina fug. El ižo del ręz se kazg kon la rika. Despugz de sięrto lisempo ęia estaba para parir. I anles k'ęia parįera demandl 40 el ręi a kign b’a iamar por kumadre. I eịa diš ke ba iamar a la ermana grande. El ręi no le refuzg. ${ }^{17}$

11 an el. Ursprung dieser offenbar Hiatus tilgenden Nebenform zu $a$ ? Vielleicht zu en, wofür an in der 379. Copla der Vida de Santo Domingo de Silos von Gonzalo de Berceo? [S. 67 der Ausg. v. Fitz-Gerald, abgedr. Zauner S. 147]. 
In dieser Nacht ging der Königssohn selber aus, um die Stadt auszuspionieren, und er kam richtig am Hause der drei Schwestern vorüber. Er sieht, dafs Licht brennt und begann durch das Schlüsselloch zu sehen und hörte, dals die drei Schwestern sprachen.

Sie hatten, um sich des Nachts nicht zu langweilen, zu sprechen begonnen. Die eine von den dreien, die älteste, seufzte und sprach: ${ }_{n}$ Ach, wenn der Königssohn mich zur Frau nähme, würde ich ihm ein sehr grofses Bett machen, dafs die ganze Welt sich hineinlegen könnte und noch Platz wäre".

Die zweite sprach: "Wenn mich der Königssohn näbme, würde ich ihm eine sehr grofse Decke machen, dafs die ganze Welt sich [damit] zudecken könnte und noch Platz wäre".

Die jüngste sprach: „Ich nicht! Ich würde ihm zwei Kinder gebären, die einen Stern und einen Mond auf der Stirne tragen."

Das alles hörte der Königssohn. Er ging nach Hause, und am Morgen liefs er die drei Schwestern holen.

Ihnen schien es, dafs der König sie holen liefs, weil sie Licht angezündet hatten. Sie waren in grofser Angst. Sie wollten nicht gehen, aber man setzte ihnen sehr zu, und sie gingen.

Der König fragte sie, was sie in der Nacht gesprochen hatten. Sie schämten sich zu sprechen. Der König drohte ihnen und sie sprachen :

Die älteste sprach: „Wenn du mich [zur Frau] nimmst, würde ich dir ein grofses Bett machen, dals die ganze Welt sich hineinlegen könnte und noch Platz wäre ${ }^{\mu}$.

Die zweite sprach: „Ich sagte, dals wenn du mich nähmest, ich dir eine sebr grofse Decke machen würde, dafs die ganze Welt sich [damit] zudecken könnte und noch Platz wäre".

Und die kleine sagte: „Wenn du mich nähmest, würde ich dir zwei Kinder gebären, die einen Stern und einen Mond auf der Stirne trügen".

Der König wollte sie auf die Probe stellen, ob sie die Wahrheit sprächen, und sagte zu der jüngsten: "Ich werde dich zur Frau nehnen, um zu sehen, ob du die Wahrheit sprichst ${ }^{*}$.

Und so geschah's. Der Königssohn heiratete die jüngste. Nach einer gewissen Zeit nahte ihr die Niederkunft. Und ehe sie niederkam, fragte der König, wen sie als Hebamme holen werde. Und sie sagte, dafs sie ihre älteste Schwester rufen würde. Der König schlug es ihr nicht ab.

ss una estreja i una luna Halbmond mit Stern, tk. jeulis (aj jyldyz), Tewfik 381, III, also wahrscheinlich türkisches Motiv. Auch die Wortstellung ist auffällig.

10 hyeron für se hyeron auch im Konstantinopler Dialekt, z. B. Wagner S. 29, V, 99.

$1 v$ le refurg und Zeile 59 le aćeto, das Akkusativ-Objekt nicht ausgedrückt. 


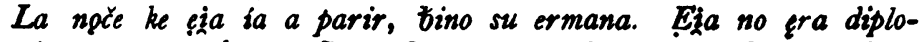
mada i gra mui selgra. Se selata muto de la ermana ke tomp 18 al fižo del ręi.

Per beggarse de ela, tome un perikiko tiko. I kuando la ręina parìg, a lugar de le dar la kriatura, eía metieg el periko en la kama. La kriatura ke tenía una luna $i$ una estrẹ̦a en el megio, enterg biba en ia buerta del ręi.

Kuando la reina demando lo ke es ke pario la ermana le respondip: 50 'No fäbles tu! No parites to kualo le prometites al rgi. Ez un per̄iko ke pariles.' - Einja estata mui espantada ke el ręi no la ẹče d'en kaza.

El ręi, komo la kería mučo, la pardong, dizięndo: 'No impqrta por esta bes. A la otra bẹs puede sęr pares to ke tu me prometites.

55 Despuęs de un año, la ręina estubo de muęto preñada $i$ el ręi le demandp a kien ta 'iamar esta bes por kumadre. Ejia respondijo: 'Para ke no tęngan sẹlo la una de la gtra, b'a iamar a mi segunda ermana'.

El ręi le aletet ${ }^{17} i$ al mizmo punto la embį̀ a ziamar. La segunda

60 ermana gra tambięn mui selgza i pqr beggarse de la rgina, tome un gatiko ciketiko $i$ fizo lo mizmo de la etra ermana. Apenas la ręina parig, la kumadre tome la kriatura, la 'nterg bita en la bugrta del riti $i$ metig el gatiko a la kama.

La rgina dentando, si parį̣ la kriatura kon la estrẹ̦a $i$ la luna 65 en el megio. - 'Estate kaiada', le respondìg su ermana, 'esla ţ̧s parites un gatiko $i$ por siguro ke el rę te ta 'Car de kaza! Tu huples mentirpza!' - La ręina ečp un suspiro $i$ diso: 'Il estaba mui sigura de lq ke le aprometía a el ręi. Komo bigne a ser ke iq para un gatiko. Está una kosa estraordinarial'

70 Despues de medila gra, el ręi entra a la odá de la reina per sabegr si ne lo engani de muęto. La kumadre le amostrq el gatiko. El ręi apenas to bido, no kižo mas saber de nada: Le dip unas kunantas parás a la ręina, la tomeq por el braso i la ečq a la kaine.

El ręi pensg $i$ dišo ęntre si: Kale sẹa ke no ęz mazdt ke io 75 tomi a esta. Agqra tq a probar a la grande $i$ samoz ber, 19 si $i$ esta ta a salir mentirqza kqmo la čika.

I ansina fü. La embig a jamar i la tubo por mužęr. La grande estata mucto alegre. Eija ia pensq ke un dia esto ba abenir ke le la 'fazer la kama.

18 tomq. Indikativ auffällig. 
In der Nacht, in der sie niederkommen sollte, kam ihre Schwester. Sie war keine zünftige Hebamme und war sehr neidisch. Sie beneidete ihre Schwester sehr, dals sie den Königssohn geheiratet hatte.

Um sich an ihr zu rächen, nahm sie ein kleines Hündchen. Und als die Königin niederkam, legte sie, anstatt ihr das Kindchen zu geben, das Hündchen ins Bett. Das Kindlein, das einen Mond und einen Stern auf der Stirne trug, vergrub sie lebend im Garten des Königs.

Als die Königin fragte, was sie geboren hätte, antwortete ihr die Schwester: „Rede nicht! Du hast nicht geboren, was du dem König versprachst. Ein Hündchen hast du geboren". - Sie war in grolser Angst, dals der König sie aus dem Hause jagen würde.

Da der König sie sehr liebte, verzieh er ihr und sprach: „Für dieses Mal macht es nichts. Ein anderes Mal gebierst du vielleicht, was du mir versprochen hast."

Nach einem Jahr ward die Königin wiederum schwanger, und der König fragte sie, wen sie diesmal als Hebamme baben wollte. Sie antwortete: „Damit sie aufeinander nicht neidisch werden, will ich meine zweite Schwester holen lassen".

Der König willigte ein, und denselben Augenblíck liefs er sie holen. Die zweite Schwester war ebenfalls sehr neidisch, und um sich an der Königin zu rächen, nahm sie ein ganz kleines Kätzchen und tat damit so, wie die andere Schwester. Kaum war die Königin niedergekommen, als die Hebamme das Kindlein nahm, es lebend im Garten des Königs vergrub und das Kätzchen ins Bett legte.

Die Königin fragte, ob sie das Kind mit dem Stern und dem Mond auf der Stirne geboren habe. - „Schweig' still! “, antwortete ihre Schwester, „diesmal hast du ein Kätzchen geboren, und sicher wird der König dich aus dem Hause jagen. Du warst [bist] eine Lügnerin!“ - Die Königin seufzte und sprach: "Ich war dessen ganz sicher, was ich dem König versprach. Wie kommt es, dafs ich ein Kätzchen gebäre. Es ist eine merkwürdige Geschichte!“

Nach einer halben Stunde tritt der König in das Zimmer der Königin, um zu hören, ob sie ihn nicht wieder betrogen hätte. Die Hebamme zeigte ihm das Kätzchen. Kaum hatte es der König gesehen, als er nichts mehr hören wollte: Er gab der Königin ein bifschen Geld, nahm sie am Arm und warf sie auf die Strafse.

Der König dachte und sprach bei sich: Es muls nicht [vom Schicksal] bestimmt sein, dals ich diese nahm. Jetzt will ich die älteste auf die Probe stellen. Wollen sehen, ob auch sie sich als Lügnerin erweisen wird, wie die jüngste.

Und so geschah's. Er liels sie holen und nahm sie zur Frau. Die älteste war sehr froh. Sie dachte sich wohl, dafs es eines Tages so kommen würde, dals sie ihm das Bett machen würde. teremos.

19 šamos, offenbar mit Aphärese aus desamos. Ebenso: ša tierẹ, šamoz 
80 La tika desmazalada estuto. Kaminando trẹs dias $i$ trẹs nptes a la fin no pudo mas kaminar. Se asento en sima de una pigdra kon el gatiko $i$ el periko, mui dezesperada. Fija tido d'en frẹnte un relumbrar de lus. Se aserkp de esta kasa, dig a la pugrta i un coban le abriq.

85 'Ke kigres en esta pra en mi kaza?', le demande el cobán. 'Ağidgate de mi, cobán!' If fug una rgina. Me deskansaré $i$ iq te t'a kontar mi triste estoria.'

Ansina fug. El cobán en sintiegndo todo lq ke le konto la regina tuto piedá de ęa $i$ le dišo: 'I in tambięn este mučo sufrięndo. Mi

90 mužger muriqg ia ai dqz años. Iq esto sọlo $i$ asolado en essta kaza. Si es ke tu estás konlęnta, io te t'a tomar kqmo una fïa. Tu tas a fazer todo lo ke ez de menester para dirizir una kaza i bamoz a biber ansina.' - ,Mui bugno', dišo la ręina.

El ręi estuto pasando buena bida kon la grande ermana. Un 95 día el estuto kaminando por su tuęrta $i$ está bį̀endo ke en un kanto

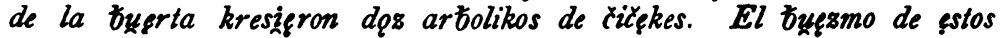
cięçkes gra tan bugno ke el ręi se aserkg a lado. Le plazian muto a el rej $i$ ningun dia no se kansata de estarlos admirando.

Loz doz arbolikos estaban en el mizmo kanto gnde la ręina enterg 100 las dęs kriaturas de la ermana cika. Ansina la ręina tübo grande espanto en bięndo el reji siempre en este mizmo lugar.

Iame una fitizera en su palasio $i$ le diso: "Il te to a dar tqdos miz biggnes, si tu pugdes fazer destatearlo a el reg ke ng se asignte mas en este lugdr.' - 'Ez mui kolak', respondiłg la fitizęra, 'iq le bp 105 a metgr grande espanto $i$ bo a kemar los drboles.'

Ansina fue. Un día ke el rej estuto asentado de lado de lqz des artolikos, pasq la fitizęra i le diso: 'Por ke razqn te asentas aki? Este byzzmo no ez bugno para tu salu. So un tósego. Sal pręsto de aiti, mi fizo. Iq nq te kijero ber mal. Eq per esto ke te esto dizigndo.

110 El ręi en sintięndo ęsto, tuto espanto i no kižo maz bęr los arbolikos. Aggra la fitizera las keme. Ma en este mizmo lugár kresigg un erimezo iertado.

Un día el cobán en ibando sus kodrẹdos en el kampo, pase por la buerta del ręi, bido el ermozo ierbado $i$ diso entre si: 'Este ier-

115 bado está mui bugno. De agera en delantre to a traiger kada dia mis kodrędos aii.'

Ansina fue. El estubo kontuneando $i$ les traia kada dia. Despuess de un mes parig un de sus kodrẹdos dos kriaturas kon la estrẹia $i$ la luna en el megio. Kuando el coban bido esto, los tomp $i$ los ito 
Die jüngste war [ganz] unglücklich. Sie wanderte drei Tage und drei Nächte, bis sie schliefslich nicht mehr konnte. Sie setzte sich auf einen Stein mit dem Kätzchen und dem Hündchen, in heller Verzweifelung. Da sah sie vor sich einen Lichtschimmer. Sie ging anf dies Haus zu, klopfte an die Tür, und ein Hirt machte ihr auf.

„Was willst du zu dieser Stunde in meinem Hause?", fragte sie der Hirt. - „Hab' Erbarmen mit mir, Hirt! Ich war einst eine Königin. Ich will mich ausruhen, und dann werde ich dir meine traurige Geschichte erzählen."

Und so geschah's. Wie der Hirt alles hörte, was ihm die Königin erzählte, hatte er Mitleid mit ihr und sagte zu ihr: „Auch ich habe grolsen Kummer. Meine Frau ist schon vor zwei Jahren gestorben. Ich bin einsam und verlassen in diesem Hause. Wenn du's zufrieden bist, will ich dich als meine Tochter annehmen. $\mathrm{Du}$ wirst alles machen, was not tut, um ein Haus in Ordnung zu halten, und so werden wir [miteinander] leben. ${ }^{*}$ - Ich bin's zufrieden ", sprach die Königin.

Der König lebte mit der ältesten Schwester glücklich und in Freuden. Eines Tages ging er in seinem Garten spazieren und bemerkt, dafs in einem Winkel des Gartens zwei blühende Bäumchen wuchsen. Der Duft dieser Blüten war so süfs, dafs der König auf sie zuging. Sie gefielen dem König sehr, und keinen Tag wurde er müde, sie zu bewundern.

Die beiden Bäumchen standen aber im gleichen Winkel, wo die Königin die beiden Kinder der jüngsten Schwester vergraben hatte. Daher geriet die Königin in grolse Furcht, als sie den König immer an dieser selben Stelle sah.

Sie rief eine Hexe in ihren Palast und sprach $2 u$ ihr: ${ }_{n}$ Ich will dir all mein Hab und Gut geben, wenn du den König davon abbringen kannst, daís er sich noch weiter an diese Stelle setzt". "Das ist kinderleicht", antwortete die Hexe, ${ }_{n}$ ich werde ihm einen grofsen Schrecken einjagen und werde die Bäume verbrennen".

Und so geschah's. Eines Tages, als der König bei den beiden Bäumchen sals, ging die Hexe vorbei und sprach: "Warum setzt du dich hierher? Dieser Duft ist nicht gut für deine Gesundheit. $\mathrm{Er}$ ist ein Gift. Geh schnell fort von hier, mein Sohn. Ich will nicht, dafs du krank wirst. Darum sage ich es dir."

Als der König das hörte, bekam er Angst und wollte, die Bäumchen nicht mehr sehen. Da verbrannte sie die Hexe. Áber am gleichen Fleck wuchs ein schöner Rasen.

Eines Tages kam der Hirt, als er seine Schafe auf die Weide trieb, am Garten des Königs vorbei, sah den schönen Rasen und sprach bei sich: „Dieser Rasen ist sehr gut. Von jetzt ab werde ich meine Schafe alle Tage hierher treiben".

Und so geschah's. Er trieb sie nun weiter jeden Tag [dorthin]. Nach einem Monat gebar eins von seinen Schafen zwei Kinder mit dem Stern und dem Mond auf der Stirn. Als der 
120 pręsto ey kasa en dizigndo a su fiža: 'Te to a kontar unza kqza de marabia. Te lo бas a kregr?' - 'De ke no?', respondíg su fiza.

'Abras a satger ke iq un dia kaminando por el palasig del rgi, bide en su buerta un tan ermezo ierbad̃o, ke me traía kada dia mis kodrẹdos a komęr de este ierbado. Despues de un mes parį̣ un kodrẹdo

125 estas dos ermozas kriaturas kon la esirẹia i la luna en el megio. Ig penst en mi $i$ diše ke ez mežor ke tu tomes al lugár de el per̃iko $i$ el gatiko ẹtoz dos cikos anğelikos $i$ ke lęz mantęngas kęmo tuz tẹras kriaturas.'

Ẹia en tiendolas tuto grande tembler en su puerpo entero $i$ diso:

130 'Esto es lo ke iq abia prometido a el ręi. Miz dos ermanas tutigeron grande sẹlo de mi $i$ por tengarse, tomaron miz bẹraz kriaturas. $i$ metiegron en mi kama el gatiko $i$ el periko. Agera las este entendįendo. El Diq. ke se begge de ẹalas komo se beygaron de mi atma!'

Despuęz de unos kuantos años, las kriaturas se fizįgron grandes 135 i se ian kada dia a kaminar por el palașio de el ręi. Un dia el ręi loz biäo de en fręnte. Los įamg $i$ se interesg mui muto en biendo ke todos dos tenian la estrẹia $i$ la luna en el megio. El ermaniko $i$ la ermanika ęran tan grasį̣zos ke 'l ręi lęz dišo: Binį̨endo

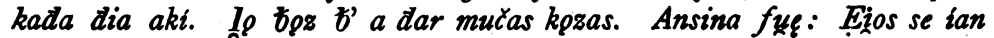

140 kada dia.

La reina, apenas lez bido, muriq del espanto i ne sabia lo ke fazęr. 'Por seguro ke sqn los fižs de mi ermana', fablo la ręina entre si, 'agora si el ręi lo ta a satęr, a mi me ta a matár!' La ręina estuto iorando del espanto, firo traier en su palasi̦o la ficizera

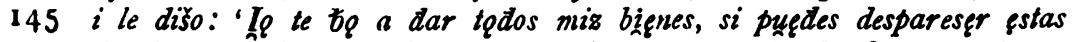
des kriaturas.' - 'NQ te siklęjes; 20 le respondịq la fixizęra, 'íq en este mizmo punto los puedo disparasęrlos. Estate en reppzo!'

Laz dqs kriaturas se estaban ięndo para en kaza. La fitizẹra ios 21 aretg $i$ les diš a la maz grande ke gra la niña: 'Me kigeres

150 fazęr un plazeriko, hanim?' - 'Si, kon muto plazęr,' respondìq la niña, 'A mi me ambezQ mi máma, ke ne refuze nunka, kuando me demandan algun plazęr. Inda mas a mužęres b̨̨̌̃žas!' - 'Mui bųno! Toma ẹste sestiko $i$ esta mantẹka! Bate por esta montaña ar̃iba! Ai taz a tęr a mi ermano i le taz a dar tqdo a ęl. Tu ermaniko ke

155 te aspẹre akil Kuando taz a tornar, tǫz baš derẹ̌o ey kaza!'

20 siklejes. Der Gleitlaut $y$ (i) zwischen $e$ und anderen Vokalen auch leonesisch-asturisch und aragonesisch-navarresisch. 
Hirt das sah, nahm er sie, trieb sie schnell nach Hause und sprach zu seiner Tochter: "Ich werde dir eine wunderbare Geschichte erzählen. Wirst du es glauben ?" — „Warum nicht?", antwortete seine Tochter.

„Du mulst wissen, dafs ich eines Tages, als ich am Palaste des Königs vorbeiging, in seinem Garten einen so schönen Rasen sah, dals ich mir meine Schafe jeden Tag zu diesem schönen Rasen auf die Weide trieb. Nach einem Monat gebar ein Schaf diese beiden schönen Kinder mit dem Stern und dem Mond auf der Stirn. Ich dachte und sprach bei mir, dals es besser ist, wenn du an Stelle des Hündchens und des Kätzchens diese beiden kleinen Engelchen nimmst und sie wie deine rechten Kinder aufziehst."

Als sie sie sah, da zitterte sie gewaltig am ganzen Körper und sprach: "Das hatte ich ja dem König versprochen. Meine beiden Schwestern waren sehr neidisch auf mich, und um sich zu rächen, nahmen sie meine rechten Kinder und legten mir das Kätzchen und das Hündchen ins Bett. Jetzt durchschaue ich sie. Gott möge sich an ihnen rächen, wie sie sich an meiner Seele rächten.

Nach einigen Jahren waren die Kinder herangewachsen, und jeden Tag gingen sie zum Palaste des Königs spazieren. Eines Tages sah der König sie vor sich. Er rief sie heran und sah mit grofsem Interesse, dafs alle beide den Stern und den Mond auf der Stirne trugen. Die beiden kleinen Geschwister waren so reizend, dals der König za ihnen sagte: „Kommt alle Tage hierher. Ich will euch viele [schöne] Sachen schenken." Und so geschah's. Sie gingen alle Tage hin.

Kaum hatte sie die Königin gesehen, als sie in Todesangst geriet und nicht wufste, was sie machen sollte. "Sicher sind es die Kinder meiner Schwester", sprach die Königin bei sich, „wenn das jetzt der König erfährt, so wird er mich totschlagen!" Die Königin weinte vor Angst, liefs die Hexe in ihren Palast holen und sagte zu ihr: "Ich will dir all mein Hab und Gut geben, wenn du diese beiden Kinder beseitigen kannst." - "Keine Sorge!", antwortete die Hexe, ${ }_{n}$ ich kann sie denselben Augenblick beseitigen. Sei ganz ruhig!

Die beiden Kinder waren auf dem Wege nach Hause. Die Hexe hielt sie an und sagle zu dem älteren, dem Mädchen: „Willst du mir einen Gefallen tun, kleines Fräulein ?" - Ja, mit grofsem Vergnügen ", antwortete das Mädchen, „mich lehrte meine Mama, dars ich niemals nein sagen soll, wenn man mich um einen Gefallen bittet. Um so mehr zu alten Frauen!“ - „Sehr schön! Nimm das Körbchen und diese Butter. Geh auf diesen Berg hinauf! Da wirst du meinen Bruder sehen und wirst ihm alles geben. Dein Brüderchen soll hier auf dich warten. Wenn du wieder zurückkommst, geht ihr geradewegs nach Hause."

21 los für las, ebenso Zeile 202 los bỉ̇o. Es schwebt etwa los fix̌os oder los ermanikos vor. Vgl, auch zu I, 9. 
Este lugar ke se fue la niña era fixizado. Fira uy kampo muito grande $i$ todo modo de persọna ke beniba por aij, se fasia trogko. $K$ yando la niña se aserkp, no atkansp a ir mas ar̃iba ke la se fiso $i$ sia lo mixmo.

160 Su ermaniko la estuto asperando. A la fin no pudo mas, se fug ey kaza, le kontg todo a su madre $i$ la fug a eykontrarla. El estuto kaminando por el mizmo lugár $i$ aritito sęrka del kampo fixizęro. Un derbis lo bido de en frente $i$ le dišo: 'Onde bas, manseto, por estos lugares ficizerros? Aki no benites por tu bį̣n sino por tu mal. Atgr165 nate pręsto de onde tu benites!"

El nino suspirq $i$ dišo: 'I mañana ke la embig una bigkža para ke le fizigra un mandadiko, i ęa inda no tígne!' - 'A', dišo el dertiš, por seguro ke tu ermana se fizo un troyko. Estáz biendo essta šara įnna de trogkos? Kada uno

170 kontigne una persona. Aki ai una prinsẹsa ke fü $i$ ẹina ficizada $i$ se fiso trogko $i$ tręnia kabaierros la binizron a buškar, 22 se fizį̧ron todos lo mizmo. Este lugar es ficizad̃o. Akabidate de aserkarte!'

Ma el niño estubo ansina de 23 triste ke suz qžos empesaron a entirse de lagrimas. 'No ipres!', le dišo el dertiš, 'ia ai remędio 175 para saltar todas estas persọnas. Estd́s bięndo at en fręnte una niña? Ésta tiegne un anižo en el dędo. Si se lo pueges kitar, tu saltas a mučas personas.' -

'Mui buçno', respondip el ermaniko. 'Mismo ke sẹa espanto, is ing me to a atrebir.' Ansina fug. El tiko se eskondìq detras de un 180 drtole $i$ kłando la nina bino para labarse, 24 kurizig el ciko, le kito el anižo $i$ empese a kor̈err.

Esta gra la fiža de un grande ręi. Apenas ke el niño le kite el anio, sia le jumg $i$ le diso: 'I a me salbates, mansęto, bęn kon mi agera en mi kazal Kuando te prezęnto kon mi padre - $A$, ne se 185 siguro, si mi padre bite! - amá in̨ kon todo bęn!"

Kundo el nino abolto la kara per la gtra parte, bido su ermana ke estata kurīiendo para abrasarlo. En fin se bezaron $i$ se fueron todos tres al palasio de la prinsesa en una kon les trenta kabaigeros ke tambinen eran desficizados.

\section{Relativsatzes.}

29 Kabajeros la binjeron Parataxe der naiven Erzählung an Stelle eines

28 ansina de triste für ansina triste wahrscheinlich unter Einwirkung des gleichbedeutenden este karar (tk. J kadar, Tewfik 259, II) de triste. 
Die Gegend aber, wo das Mädchen sich hinbegab, war verhext. Es war ein sehr grolses Feld, und jedermann, der dorthin kam, wurde in einen Baumstamm verwandelt. Wie das Mädchen näher kam, konnte sie [plötzlich] nicht mehr weiter, denn schon wurde sie in der gleichen Weise verwandelt.

lhr Brüderchen wartete auf sie. Schliefslich dauerte es ihm zu lange, er ging nach Hause, erzählte alles seiner Mutter und ging ihr [der Schwester] entgegen. Er ging zu dem gleichen Orte und kam an das verhexte Feld. Ein Derwisch sah ihn vor sich und sagte $z u$ ihm: "Wohin gehst du, junger Mann, in dieser Zaubergegend. Hierher kamst du nicht zu deinem Glück, sondern zu deinem Unglück. Kehre rasch wieder um, woher du kamst! ${ }^{4}$

Der Knabe seufzte und sprach: „Ich kam meine Schwester zu suchen seit [heute] Morgen, wo sie eine Alte schickte, ihr einen kleinen Gang zu machen, und sie kommt immer noch nicht!" ${ }$ Ach", sagte der Derwisch, ${ }_{n}$ sicher ist deine Schwester in einen Baumstamm verwandelt. Siehst du diesen Wald voller Bäume? Jeder [Baum] schliefst einen Menschen ein. Hier ist eine Prinzessin, die auch verzaubert und ein Baumstamm wurde, und dreifsig Ritter kamen sie zu holen, sie wurden alle das gleiche. Dieser Ort ist verhext. Hüte dich, näher zu kommen!

Aber der Knabe wurde so traurig, dafs seine Augen begannen sich mit Tränen zu füllen. "Weine nicht! ! , sagte der Derwisch zu ihm, nes gibt noch ein Mittel, um alle diese Leute zu erlösen. Siehst du hier vor uns ein Mädchen? Die trägt einen Ring am Finger. Wenn du ihn ihr wegzunehmen vermagst, erlöst du viele Leute."

"Sehr gut", antwortete das Brüderchen, nmag es auch gefährlich sein, so will ich es dennoch wagen". Und so geschah's. Der Kleine versteckte sich hinter einen Baum, und als das Mädchen kam, sich zu waschen, stürzte der Kleine auf sie zu, nahm ihr den Ring weg und wollte davon laufen.

Es war die Tochter eines grofsen Königs. Kaum hatte ihr der Knabe den Ring weggenommen, als sie ihm nachrief und zu

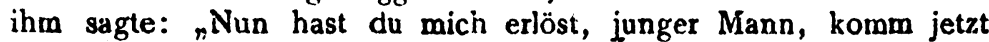
mit mir 20 mir nach Hause. Wenn ich dich meinem Vater vorstelle ... ach, ich weifs nicht einmal sicher, ob mein Vater lebt!... aber komm trotz alledem!"

Als der Knabe den Kopf nach der anderen Seite wandte, sah er seine Schwester, die auf ihn zulief, um ihn zu umarmen. Schliefslich küfsten sie sich und begaben sich alle drei zum Palast der Prinzessin, zusammen mit den dreifsig Rittern, die ebenfalls entzaubert waren.

24 labarse. Motiv einer t'berraschung des Mädchens im Bade ron des Erzäblerin anscheinend vergessen. 
Kunando el ręi tid̄o su fïa, tubo una grande alegria.' Le demando qnde estuto fin a ẹstos todos 25 años. La prinsẹsa le konto todo $i$ le prezento al mansẹto ke la salto.

El ręi per engrasiarlo le dišo ke lo kigere tomar pgr įqrno a le 'mpesg a informarse 26 de ke famiä a apartįnne. El nino kqmo ia gra

195 maz grandeziko, supo la triste 'stqria de su madre. Kuando el ręi sintip tydo esto, le dišo a el niño: 'Una bẹs ke the salbates a mi fixa, ig te 't' a fazer una grande buendá.'

En el mizmo punto intite en su palasio a la ręina i a el ręz a pranso. Fiso eskonder loz doz ermanikos detraz de una pueqta. Se

200 fuęron loz doz ręies en qtra odá. Le konto tod̄o el pasaže i le amostrq suz tęras kriaturas.

Kunando el ręi loz 21 bid̈o, loz abrase $i$ les demandg onde está suz madre. Las kriaturas le kontaron tqdo $i$ kuando el ręi supo ke su prima mužgr bite inda, lez dišo a las kriaturas: 'Asperándobos akí.

205 Agqra bamoz a ir teälos tres a tomar a máma.' Las kriaturas esiutieron mui alegres en supiegndo ke sus padre es el ręi.

Agora el un ręi le diso a el gtro: 'Komo moz tamoz a beygar d'esta kruęla persọna?' - ' $A$, ęz mui kolái, no tenemos $k$ ' a demandarle 27 an eịa mizmo.' 28 Ansina fug: Le demandaron a la ręina: 210 'Ansina $i$ ansina una persọna ke faze mal a el ręi ke detẹmoz 29 de faserle?'

La reina responding: ' $A$, una persona ke faze mal a el ręi merese malado 30 i tomad̃o el puerpo kortado en mucos pedasos $i$ metido kada pedaso a las puertas de la sivdá entera.'

215 'Mui buęnol', respondį̀ el ręż, 'ẹsto está una mui buęna pensada para til' En el mismo punto iame uno de suz mosos. La degojaron $i$ metigron un pedaso de karne en kada pugrta de la sivdd.

El rei tome suz dos kriaturas $i$ se fue a tomar a su prima $i$

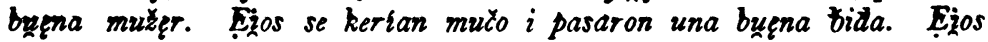
220 saltados i mozgtros dukados!

25 fin a estos todos años. Kontaminiert aus fin a este año (oder dia) und estos todos años.

${ }_{80}$ le 'mpesp a informarse. Neben empesp a informarse schwebte wohl le empesp a demundar vor.

21 no tenemos k'a demandarle Gallizismus. 
Als der König seine Tochter sah, freute er sich sehr. Er fragte sie, wo sie die ganzen Jahre über gewesen sei. Die Prinzessin erzählte ihm alles und stellte ihm den Jüngling vor, der sie erlöst hatte.

Um es ihm zu vergelten, sagte der König, dals er ihn zum Eidam nehmen wolle, und begann sich zu erkundigen, was seine Familie wäre. Der Knabe, der schon ziemlich grols war, wufste die traurige Geschichte seiner Mutter. Als der König das alles hörte, sagte er zu dem Knaben: „Da du meine Tochter erlöst hast, will ich dir einen grofsen Dienst erweisen."

Denselben Augenblick lud er die Königin und den Könjig zu Mittag in seinen Palast. Die Geschwister liefs er hinter einer Tür verstecken. Die beiden Könige gingen in ein anderes Zimmer. Er [der Gastgeber] erzählte ihm [dem Gaste] alles, was geschehen war, und zeigte ihm seine rechten Kinder.

Als der König sie sah, kürste er sie und fragte, wo ihre Mutter wäre. Die Kinder erzählten ihm alles, und als der König vernahm, dafs seine erste Frau noch am Leben sei, sagte er zu den Kindern: ${ }_{n}$ Wartet hier. Jetzt wollen wir alle drei die Mama holen gehen." Die Kinder freuten sich -sehr, als sie erfuhren, dars ihr Vater der König sei.

Da sagte der eine König zum andern: "Wie wollen wir uns an dieser grausamen Person rächen ?" - ${ }^{\circ} \mathrm{Oh}$ ! das ist ganz leicht, wir brauchen sie nur selber $z \mathfrak{u}$ fragen." "Und so geschah's. Sie fragten die Königin: „Eine gewisse Person, die dem König übeltut, was sollen wir ihr tun?"

Die Königin antwortete: „Oh, eine Person, die dem König übeltut, verdient, dafs man sie totschlägt und inr den Leib in Stücke schneidet und die Stücke alle vor die Tore der ganzen Stadt legt."

„Sehr wohl!", antwortete der König, „das ist ein sehr guter Gedanke für dich!" Im selben Augenblick rief er einen von seinen Dienern. Sie richteten sie hin und legten ein Stück Fleisch vor jedes Tor der Stadt.

Der König nahm seine beiden Kinder und holte seine erste und gute Frau. Sie liebten sich sehr und lebten sehr glücklich miteinander. Sie sind erlöst, und für uns Dukaten!

28 mizmo für mizma auffällig.

so deter de, sp. zu allen Zeiten häufig, nach Analogie von hater de. Vgl. Cuervo $D$. II deter $S .80 \%, 2$. Spalte $\eta$

${ }_{80}$ merese matado. Vgl. Anm. 10 und deutsch: "Du gehörst auf die Finger geklopft". Rest des Absatzes anakoluthisch. 


\section{Zweiter Teil : Grammatik. \\ Erster Abschnitt. \\ Lautlehre.}

8 I. Akzent. I. ğudį̣ asp. im Reim mit prometip belegt., [Proverbios morales del Rabbi don Sem Tob, Men. Cid I, S. I67 zitiert.]

$D_{\text {iq }}$ ist jsp. Neubildung nach dem asp. belegten Plural Digs (vgl. Hanssen S. 1 24, Wagner \& 2).

2. máma [III, 15I und 205] asp. und auch amerikanisch (Cuervo, Ap. \$6r).

\section{Vokale.}

§ 2. Betonte Vokale. Die Ưberdiphthongierung von betontem und vortonigem $e$ und 0 , für das Aragonesische und Leonesiche von Hanssen (S. 20), für den Konstantinopler Dialekt von Wagner (\$3) festgestellt, findet sich auch salon. kizerian III, 25; byenda III, 197.

§ 3. Unbetonte Vokale. 1. e > a. asperar ist bereits asp. Zur Erklärung von ambezar (für embezar $<$ invitrare) ist auf ambidos aus invilus hinzuweisen (zu letzterem Zauner S. 24).

2. e $>\mathbf{i}, 0>$ u. Das Schwanken im Gebrauch von vortonigem $e$ oder $i$, bezw. o oder $u$ findet sich auch im Salonikier Dialekt. Mit Unrecht hat man diese Eirscheinung auf die Raschischrift zurückführen wollen, die für $e$ und $i$ einerseits und $o$ und $u$ andererseits nur je ein Zeichen besitzt (Lamouche S. 975). Abgesehen davon, dafs die gleiche Erscheinung sich auch im Mutterlande findet, scheint es auch mit den Prinzipien der allgemeinen Sprachwissenschaft kaum vereinbar, der Schrift, und zumal der so selten gebrauchten Raschischrift, einen derartigen Einflufs zuzuschreiben.

Beispiele: $e>i$ : dizia II, 1; fitisera III, 102; sigura III, 8, aber auch seguro III, $142 ;$ siffer I, 6 . $0>u$ : kurigron I, $5 ;$ kutizara III, I8; kumadre III, 40.

§ 4. Hiatus und Synalöphe. I. Unbetontes $i$ und $e$ vor oder nach anderen Vokalen erhalten konsonantischen Charakter, sofern es die umgebende Konsonanz zuläfst.

Beispiele: gudì , metizeron, ręina, fue, pugrta. Aber: kriatura, kruqla, sufrigndo.

2. Die bei schnellem Sprechen auftretenden Verschleifungserscheinungen sind bereits von Wagner (\$19) festgestellt und klassifiziert worden. Ich gebe daher nur einige Beispiele aus den Texten :

a) gleiche Vokale: le 'mpese a III, 194; le ia 'fazer III, 79; triste 'storia $a$ III, 195. 

III, 57 .

b) ungleiche Vokale: k' a III, 208; fin' a III, I9I; b' a jamar

c) auslautendes $a$ und $0:$ la 'nterq III, 62; te ta 'tar III, 66.

Indes findet sich salon. gelegentlich keine Synalöphe, wo sie asp. ganz gewöhnlich ist:

So hört man öfter de el (III, 135 de el rẹi) und sehr häufig a el: II, 30 a el pmbre, a el ręi III, 08, 97, a el niño III, 196.

\section{Konsonanten.}

\section{Labiale.}

§ 5. b und v. Bekanntlich unterscheidet das Jsp. noch wie das Asp. $b$ und $v[\hbar]$. Im allgemeinen stehen salon. die Laute in den gleichen Fällen wie im Konstantinopler Dialekt (zu diesem vgl. Wagner § 22).

a) intervokales $b$ wird $b$; satidas, abia, mansẹto, estaban. Ausn.: $\alpha$ ) anlautendes $b$ im Innern einer Wortgruppe: mui $b i b a$, la bos; $\beta$ ) anlautendes, durch Wortkomposition intervokal gewordenes $b$ : abašo, aboltar, abrasar; $\gamma$ ) intervokales $b$ in Fremdwörtern: moabęt, $s a b a$, die die Erhaltung des $b$ dem geminierten $b$ ihres Etymons verdanken könnten, es heifst aber auch ebenso cobin, wo pers.-tk. nur ein $b$ zu Grunde liegt.

b) $b$ zwischen Vokal und $l$ oder $r$ wird ebenfalls zu $b$ : $i \varepsilon r t a$, ártole, fatlar, palatra.

c) anlautendes asp. $b$ bleibt jsp. erhalten: $b u \varepsilon n o, b i \xi n, b q s$.

d) Ebenso hat sich asp. $b$, das durch Dissimilation aus lat. $v$ hervorgegangen ist, jsp. erhalten: bito, bitgr, bolter, (bqto).

e) labiodentales $v$ an Stelle des bilabialen $b$ findet sich in bivda, sivdó usw. (vgl. Wagner, Revue de Dial. Rom. I, 495).

f) $b$ und $v$ nach $n:$ In Saloniki heilst es wie in Konstantinopel embiar, ambezar. Indessen hört man invitar (III, 198), was ein Italianismus sein könnte, und niemals habe ich den Übergang von 6 zu $b$ nach $n$ an der Wortgrenze beobachtet: en biqzndo III, 137 .

§6. f und h. $f$ hat sich salon. im allgemeinen erhalten. Manchmal, aber dann nur vor u\& tritt dafür $h$ auf, doch schwankt der Gebrauch so stark, dafs ein und dieselbe Person bald $f$, bald $h$ spricht. III, 26.

Beispiele : fuq III, 86, II, 12, III, 77; huptes III, 67, hiuqron

Auch in Üsküb und Monastir herrscht $f$ vor. Die bulgarischen Juden indes, z. B. die Sofioter, gebrauchen die $f$-losen Formen. Sie stehen aus diesem Grunde, da man die heutige mutterländische Aussprache kennt, in dem Rufe, ein feineres Spagnolisch zu sprechen, das zur Nachahmung reizt. Die salon. 
$f$-losen Formen, deren Zahl gering ist, konnten sich mithin auf gelehrten Einflưs zurückführen lassen (anders Lamouche S. 979). Gegenüber asp. fermoso heilst es auch salon. nur ermpso.

\section{Dentale.} zurück.

\$ 7. s und z. I. Jsp. $s$ geht auf $\varepsilon$, ss und anlaut. $s$ im Asp.

Beispiele für intervokales $s$ : abrasar, alkansar, asentar, aserkar, empesar, eygrasiar, grasigzo, katesa, karapusa (aber asp. carapuza), kresę, mansęto, meresęr, nasęr, palasĩo, pasar, pedaso, pensar, prinsęsa, resibir.

Bei schnellerem Sprechen beobachtet man häufig die Vokalisierung von auslautendem $s$ vor vokalischem oder stimmhaften konsonantischem Anlaut.

Beispiele: buenos de I, I; ningunos ne moz tan III, 8; abraz a sabęr III, I 22; baz a ţr III, I54; tręz bezez en alto II, 10.

2. Jsp. $z$ entspricht dem in asp. Handschriften $z$ geschriebenen und vermutlich $d z$ gesprochenen Laut, sowie asp. intervokalem $s$.

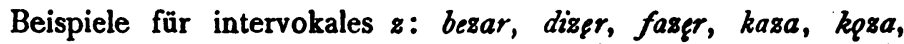
plazer, prezentar, repqso, sowie die Endung gzo (ermpso, grasịpso usw.).

§ 8. d. a) intervokales $d$ und $d$ zwischen Vokal und $r$ wird $d$.

Beispiele: ğudip, kodredos, padre, puede.

Bei rascherem Sprechen unterliegt auch anlautendes $d$ im Innern einer Wortgruppe nach vokalischem Auslaut diesem Wandel.

Beispiele: le dio II, 4; kada dia III, 140.

b) auslautendes $d$ ist salon. geschwunden: berdd, sivdd, salú. Auch Fremdwörter zeigen diesen Schwund: alhd (Sonntag) arabisch

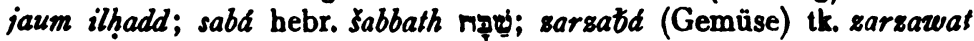
سبروات Tewfik 178, III.

§ 9. n. Im Innern einer Wortgruppe geht auslautendes n salon. vor labialem Anlaut nicht in $m$ über.

Beispiele: Eran bugnos I, 1; tan bugno III, 97.

Palatale.

$\S$ 10. g. $g$ findet sich salon. nur nach $n(y)$. In allen andern Stellungen ist es, abweichend vom Konst. Dialekt, zu $g$ geworden.

Beispiele: beggar; aber: gritar, ğugando, aggra, siguro, negro.

§ I1. i, $\check{z}, \check{\mathbf{g}}$. Die asp. Orthographie bietet vielfach keine sicheren Kriterien dafür, welcher dieser drei Laute jeweils den Schriftzeichen entspricht. Der jsp. Lautstand erlaubt hier also Rückschlüsse auf die asp. Aussprache (vgl. Wagner $\$ 37$, Zauner $\S 63$ ). 
Charaxteristuk LES JUDENSPAN. DIALEKTS VON SALONIKI. 677

\begin{tabular}{|c|c|c|c|}
\hline Beispiele: & 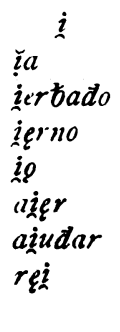 & $\begin{array}{l}\quad \text { ž } \\
\text { dirižir } \\
\text { bį̨žo } \\
\text { fïn } \\
\text { inteližęnte } \\
\text { kutižar } \\
\text { mežgr } \\
\text { mužęr } \\
\text { pasaže }\end{array}$ & $\begin{array}{l}\quad \stackrel{g}{g} \\
\text { anğgliko } \\
\text { ğudį̀g } \\
\text { ğugar } \\
\text { pgğo }\end{array}$ \\
\hline
\end{tabular}

$\S$ I 2. ¿̌. $\xi$ entspricht $\operatorname{dem} x$ der asp. Hss.

Beispiele: dišo, abašo, pdšaro.

Vor $k$ geht asp. $s$ jsp. in $s$ über. Die Anfänge dieser Palatalisierung gehen ins Asp. zurück, doch ist sie im Jsp. verallgemeinert worden. Bsp.: buškar, kaškar usiv. (vgl. Wagner § 31 ).

$\S$ 13. 11 . $l l$ ist $z u ~ i$ geworden. Nach $e$ and $i$ ist dieses oft kaum hörbar.

Beispiele: capęo und capęio II, 23 und 14; marabižado II, 2 I ; marabia III, 121 ; aji III, IO9; ai III, 153.

Die Entwicklung von $l l \mathrm{zu} i$ ist, wie Wagner $\$ 38$ bemerkt, noch nicht asp.

§ 14. Konsonantenzuwachs. I. n: ansina asp., wahrscheinlich andalusisch (vgl. Espin osa, Revue de Dial. Rom. I, I83 Anm. 4).

2. $\delta$ : An Stelle von anlautendem $u \varepsilon$ hört man oft $b_{k \varepsilon}$.

Beispiele: ųrka III, 6; aber buerta III, 95; бu६zmo III, 108.

Dieses $t$, das sich infolge des frikativen Charakters des leicht einstellt, bildet das Gegenstück zu dem $g$, das im Konstantinopler Dialekt vor anlautendem ye erscheint. Vgl. über dieses Wagner $\S 40$, S. 113 , Subak, Judsp. S. 159, Subak, Sal. S. 10, § 34.

3. $r$ : delantre schon asp. belegt (Wagner $\$ 41$ ).

\$ ij. Konsonantenwechsel. 1. $m>n$ : mps, mosptros, mųstro sind dialektisch und vulgärspanisch, vielleicht nach Analogie von me gebildet.

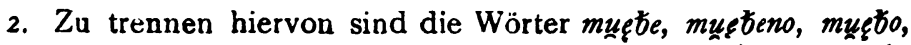
mųra, mųs und bimuęlo (aus buñuelo, Wagner $\$$ I5), die, wie bereits Lamouche gesehen hat (S. 982), ihr $m$ der 'nalure labiale de l'u consonne du groupe $u \ell^{\prime}$ verdanken (vgl. auch Subak, Verbum S. 325, Anm. 1).

dezmudarse für desnudarse 'sich ausziehen' scheint von mudar oder ezmudar beeinflufst zu sein. Übrigens notiert Subak deznudo (Sal. S. 16, Anm. I).

§ 16. Assimilation. puefpo für kyerpo recht häufig (vgl. Wagner $\S 44$ a).

\$17. Metathese. 1. Konsonanten: probe.

2. Vokale: kontunear, luęnga. 


\section{Zusammenfassung.}

Phonetisch trägt der Dialekt von Saloniki im allgemeinen die gleichen Züge wie der von Konstantinopel. Abweichend ist vor allem die Bebandlung von $f$ und $g$, von auslautendem $d$ und anlautendem uq.

\section{Zweiter Abschnitt. \\ Wortlehre. \\ Kapitel I: Formenlehre.}

A. Verbum. Verbums.

Die Darstellung umfafst nur die Formen des regelmä/sigen

§ 18. Indikativ Präsentis.

\begin{tabular}{lll}
\multicolumn{1}{c}{ I } & II & III \\
tomo & bendo & resibo \\
tomas & bendes & resites \\
toma & bęnde & resibe \\
tomamos & bendẹmos & resibimos \\
tomás & bendęs & resitiš \\
toman & tęnden & resiben
\end{tabular}

Die Palatalisierung des $s$ der 2. Plur. infolge des voransgehenden $i$ und des letzteren Schwund ist phonetisch leicht verständlich. Auf die Analogie in der Entwicklung von sęjs zu jsp. sps hat bereits Subak aufmerksam gemacht.

§ 19. Konjunktiv Präsentis.

\begin{tabular}{lll} 
I & \multicolumn{1}{c}{ II } & III \\
tome & benda & resiba \\
tomes & bęndas & resibas \\
tome & benda & resiba \\
tomemos & tendamos & resibdmos \\
tomęs & bendds & resibaj \\
tQmen & tęndan & resiban
\end{tabular}

$\S 20$. Indikativ Imperfekti.

\begin{tabular}{|c|c|c|}
\hline $\begin{array}{l}\text { I } \\
\text { tomaba } \\
\text { tomabas } \\
\text { tomaba } \\
\text { tomabamos } \\
\text { tomabas } \\
\text { tomaban }\end{array}$ & $\begin{array}{l}\quad \text { II } \\
\text { bendta } \\
\text { bendias } \\
\text { bendía } \\
\text { bendiamos } \\
\text { bendias } \\
\text { bendian }\end{array}$ & $\begin{array}{l}\quad \text { III } \\
\text { resibia } \\
\text { resitias } \\
\text { resibia } \\
\text { resibiamos } \\
\text { resitias } \\
\text { resibian }\end{array}$ \\
\hline
\end{tabular}

§ 21 . Konjunktiv Imperfekti durch das Plusquamperfektum verdrängt (s. $\S 38)$. 
§ 22. Futur und Konditionel sind ziemlich selten.

Beispiele für Futur: abrás III, 122; deskansaré III, 86.

Die gesprochene Sprache zieht meist die umschreibenden Formen vor (s. \& 46, 2).

§ 23. Plusquamperfektum.

\begin{tabular}{|c|c|c|}
\hline \begin{tabular}{l}
\multicolumn{1}{c}{ I } \\
tomara \\
tomaras \\
tomara \\
tomáramos \\
tomáraš \\
tomaran
\end{tabular} & 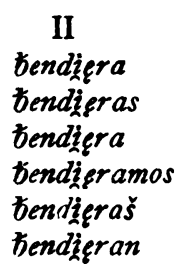 & 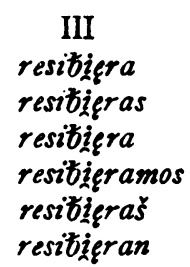 \\
\hline
\end{tabular}

§ 24. Präteritum.

$\begin{array}{lll}\quad \text { I } & \text { II } & \text { III } \\ \text { tomi } & \text { bendi } & \text { resibi } \\ \text { tomates } & \text { bendites } & \text { resibites } \\ \text { tomg } & \text { bendig } & \text { resibil } \\ \text { tomamos } & \text { bendimos } & \text { resibinios } \\ \text { tomates } & \text { bendiles } & \text { resibites } \\ \text { tomaron } & \text { bendigron } & \text { resibieron }\end{array}$

Zur Erklärung der I. Sing. hat bereits Subak (Verbum S. 327) auf das Paradigma von dar hingewiesen. Im Usküber Dialekt ist das $i$ bereits auch in die I. Plur. gedrungen (also tomimos), ein bedeutsamer Schritt auf dem Wege zur Angleichung des Prät. I an die II. und III. Konjugation.

Die 2. Sing. führt Menéndez Pidal (Man. S. 104, nach ihm Lamouche S. 985) auf vulgärkastilianische Formen auf -astes und -isles zurück, woraus mit Dissimilation -ates und -ites.

Bedenklich erscheint es, mit Lamouche die 2. Plur. aus asteis bezw. isteis zu entwickeln, da das Asp. nur die Endungen -astes und -istes kennt. Die Pluralendung auf -tes wird vielmehr eine jsp. Neubildung nach Analogie des Präsens und Imperfektums sein, die um so eher begreiflich scheint, als -astes und -istes ja wahrscheinlich zunächst auch die Endungen der 2. Sing. waren und sich somit das Bedürfnis nach Differenzierung geltend machen mulste.

§25. Imperativ.

\begin{tabular}{lll} 
I & \multicolumn{1}{c}{ II } & \multicolumn{1}{c}{ IIt } \\
toma & tende & resite \\
tomamos & tendemos & resibimos \\
tomando & tendįendo & resibitendo
\end{tabular}

Die 2. Plur. lautet also wie das Gerundium. Subak erklärt die merkwürdige Homonymität wie folgt (Sal. S. 16): 
ndisilde, 'sagt ihm', aber schon diziendome 'sagt mir' und so stets Gerundium statt des Imperativs 5, weil in -ed $+10>$ eldo, $-a d+l o>-a l d o,-i d+l o>i l d o$ milsverständlich noch einmal $-l o$ angehängt wurde, $\perp$ ldolo wie pindola ${ }^{1}$ zu ${ }^{\prime}$ ndolo dissimiliert, dann anscheinendes Gerundium von -ar und viendo $\mathfrak{u}$. ä. verallgemeinert und auch -me usw. angehängt wurde."

Die Subaksche Erklärung dürfte jedem Deutungsversuche überlegen sein, der in der fraglichen Form ein elliptisch gebrauchtes oder in imperativischer Funktion stehendes Gerundium 2 sehen wollte, da sie von der erklärungsbedürftigen Form selbst ausgeht und so auch der Frage gerecht wird, warum gerade diese und keine andere Person des Imperativs durch das Gerundium verdrängt wurde. Zweifelhaft mag nur die Begründung der Dissimilation erscheinen. Sollte man die Natur des ${ }_{n} l^{\mu}$ in -ldo verkannt haben, während man sie in -lde, wie das von Subak angeführte dizilde zeigt, noch versteht? Anders stände es, wenn die nur vorausgesetzten Formen auf - - ddolo etwa aus dem Konstantinopler Dialekt, wo der Imperativ auf -ad noch volle Lebenskraft besitzt, wirklich belegt wären. So erscheint es mir glaubhafter, dafs die Dissimilation von den Verben auf -lar, -ldor und -ltar ausgegangen ist, wie: aboltar, arreglar, calcular, consultar, contemplar, fablar, meldar ('lesen'), ocultar, señalar. Den Anfang könnte das sehr häufig gebrauchte und zur Dissimilation geradezu herausfordernde 'meldaldo' gemacht haben.

$\S 26$. Infinitiv.

$\begin{array}{ccc}\text { I } & \text { II } & \text { III } \\ \text { tomar } & \text { bender } & \text { resitir }\end{array}$

§ 27. Partizipium. I. Das Partizipium Präsentis kommt in der gesprochenen Sprache nicht mehr vor. Es wird durch das Gerundium ersetzt.

2. Das Partizipium Perfekti:

$\begin{array}{ccc}\text { I } & \text { II } & \text { III } \\ \text { tomado } & \text { tendido } & \text { resitido }\end{array}$

1 Gemeint anscheinend campidanisches pindola < pilula (M.-L. Wb. 6507).

2 Ein solches findet sich im heutigen Volksspanischen, wie die nach. folgenden zwei mir von Herrn Dr. Wagner gütigst mitgeteilten Beispiele zeigen. Bezeichnenderweise tritt es aber dann unterschiedslos für alle Personen und Numeri ein: ¿ Donde podriamos ir a dormir $P$ le pregunto Manuel. - Vamos a las cuevas de la Montaña - contestó el Bisco - Pero, ¿alld se podrá entrar P - Si, si no hay mucha gente. - Entonces, andando. Pio Baroja, La Busca, S. 270. Vaya, vaya, ya se ha callado usted; si no, me levanto y le dejo en poder de la madre, que se encargard de ponerle menos alegrito. iNo, por Dios! - Pues, callando. - Digame usted como se llamaba antes de ser religiosa. - etc. - Palacio Valdés, La Hermana San Sulpicio, ed. Nelson, Paris S. 58. 
CHAKAKTERISTIK DES JUDENSPAN. DIALEKTS VON SALONIKI. 68I

§28. Gerundium.

$\begin{array}{ccc}\text { I } & \text { II } & \text { IlI } \\ \text { tomando } & \text { tendiendo } & \text { resibigndo }\end{array}$

Das Gerundiưn der Verben mit besonderem Perfektstamm wird gelegentlich von diesem gebildet, eine auch in den spanischen Dialekten begegnende Erscheinung (Zauner $\$ 4$ b $\vartheta$ ).

Beispiel: supigndo III, 206.

B. Nomen.

$\S$ 29. Adjektiv. Einige Adjektiva, die sp. einer Endung sind, bilden jsp. ihr Femininum auf $-a$ (vgl. Wagner $\$ 63$ ).

Beispiel: kruęla III, 208. .

\section{Pronomen.}

$\S$ 30. Personalpronomen. I. os an Stelle von bos ist nicht mehr ins Jsp. gedrungen.

Beispiel: fuíendobos I, 8.

2.'Für asp. gelo steht dagegen bereits selo.

Beispiel : si selo pugdes kitar III, 176.

§ 31. Relativpronomen. Für qual heirst es wie im Asturischen und Aragonesischen kualo, kuala (vgl. Hanssen S. 181, Wagner § 105).

\section{Kapitel II: Wortbildung.}

$\S 32$. Nominalableitung. Überaus häufig ist der Gebrauch des Diminutivsuffixes $-i k o$, das auch an türkische Wörter tritt.

Beispiele: azniko, ermaniko, gatiko, mandadiko, per̈ıko, plazeriko.

Neben -etiko (tiketiko III, 61) aus ittu + iccu, findet sich -eziko:

Beispiele: grandeziko III, 195; gazeziko III, 7; fezezika II, 10. Man wird hierin wohl issu + iccu zu sehen haben, dessen lautgesetzliche Form -esiko der Konstantinopler Dialekt erhalten hat. -eziko für -esiko erklärt sich durch die salon. sogar in der Wortgruppe (vgl. $\& 7$ бaz a ber usw.) zu beobachtende Tendenz, intervokales $s \mathrm{zu}$ vokalisieren.

§ 33. Verbalableitung und Komposition. 1. Das Jsp. hat eine grofse Zahl türkischer Verben eingebürgert, indem es an vokalischen Stamm -dear, an konsonantischen -ear hängte (vgl. Wagner § 165).

Beispiele: siklęar sykyl-mak, ağidẹar ağy-mak.

2. Viele asp. mit dem Präfix a gebildete Komposita haben sich jsp. erhalten, während sie nsp. untergegangen sind:

Beispiele: aprometir, arogar, atornar und vielieicht akatidar. 


\section{Kapitel III: Wortschatz.}

8 34. Was Wagner über den Wortschatz des Konstantinopler Dialekts sagt ( $\$$ 119-181) gilt im wesentlichen auch für den Salonikier:

Eine grofse Zahl altspanischer, im Mutterlande verklungener Wörter haben sich jsp. erhalten. (Beispiele aus den Texten: aggra, ambezar, ansina, ar̈ibar, asendę, delantre, inda, kale, karapusa, pressto.) Die portugiesischen Elemente (burako?, akatidur?), die italienischen (atetar, ma?, pranso) und die griechischen (Beispiel: lagna von $\lambda \alpha^{\prime} \chi \alpha \nu \alpha \mathrm{Kohl}$ ) sind an Zahl gering und lassen sich infolge des Mischcharakters sämtlicher Balkansprachen selbst nicht immer mit Sicherheit nach ihrer Nationalität bestimmen. Die hebräischen Elemente (sabá, kal, mazál, demazalad̈o) sind mit wenigen Ausnahmen auf den religiösen Begriffsbereich beschränkt. Auch die französischen Elemente sind, soweit es sich wenigsténs um volkstümliches Jsp. handelt, nur in geringer Zahl vorhanden.

Indes ist für den Salonikier Dialekt eine grolse Einschränkung zu machen: Die türkische Sprache spielt hier bei weitem nicht die beherrschende Rolle. $\mathrm{Zn}$ statistischen Untersuchungen reicht das von mir gesammelte Material nicht aus. Doch genügt ein flüchtiger Vergleich der vorliegenden mit den Wagnerschen Texten, um zu erkennen, wie sehr die türkischen Elemente sich im Salonikier Dialekt in der Minderheit befinden.

Die türkischen Fremdwörter sind, wie Wagner gezeigt hat (\$§ 146-1 48), in der vulgärtürkischen, durch jüdische Aussprachebesonderheiten noch weiter veränderten Lautform ins Jsp. gedrungen.

\section{Kapitel IV: Gebrauch der Wortformen.}

\section{A. Vexbum.}

Tempora.

§ 35. Präsens. Wie im Konstantinopler Dialekt (Wagner $\$ 58)$ findet sich auch salon. in Nebensätzen oft das Präsens, wo die Consecutio temporum ein Tempus der Vergangenheit verlangt.

Beispiele: nasen II, 2; ambezan II, 3; fable II, 5; bite III, 204; es III, 206.

In den gleichen Zusammenhang gehört der Gebrauch des Präsens in der Formel es ... ke, wenn logische Schärfe des Ausdrucks ein Tempus der Vergangenheit erwarten lärst (vgl. hierzu Tobler, V. B. II, 12, Weigert S. 28, M.-L. III, § 57 i).

Beispiele: si es ęia II, 2I; ke no es el Diq II, 27; ke es el Dip II, 17; kien es II, 24 .

Indes scheint die Formel überhaupt ein Gallizismus zu sein.

Präsens historicum begegnet in den Texten nicht selten.

Beispiele: estd tikndo III, 95; to ke ai lus III, 10. 
$\$$ 36. Imperfektum. Das Imperfektum steht wie asp. gelegentlich im Sinne des Plusquamperfektums (Zauner § 182).

Beispiele: tomatan a fatlar III, 13; estata sintį̨ndo III, 22.

Wir finden es ferner in der Protasis der Bedingungssätze: si el fizo del ręi me tomata III, 14 und 17.

Auch in der Apodosis findet es sich gelegentlich, doch ist hier die Wortgruppe ia $+a+$ inf. üblicher.

Beispiele: iq le fazia an

§ 37. Praeteritum. Das Praeteritum steht wie asp. oft im Sinne des Plusquamperfektums (Wagner $\S 53$, Hanssen $\S 34,7$ ).

Beispiele: a) mit apenas eingeleirete Hauptsätze: apenas tida II, 13; apennas la ręina parig III, 61; el ręi apẹnas lo bido III, 72; la rgina apenas lqz bido III, 141 .

b) Konjunktionalsätze: apẹnas ke el niño le kito III, 182; pqrke asindieron lus III, 24.

c) Relativsätze: ke el ręi le eykomendo II, 6; ke le ambesp II, 21 ; ke parig. III, 49; onde la ręina enterg III, 99.

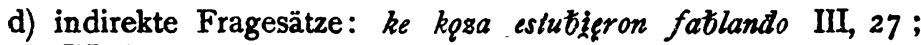
si parkig III, 64 .

§ 38. Plusquamperfektum. Das Plusquamperfektum hat jsp. seinen eigentlichen Geltungsbereich völlig verloren. Es hat die Funktionen eines Konjunktivs der Vergangenheit übernommen und den Konjunktiv auf -sse verdrängt (Wagner $§ 57$ ).

Beispiele: antes $k$ giza parigera III, 39; para ke le fizį̧ra III, 167 ; ke todo el mundo se ečara III, 16; ke ubiegra lugar III, 16, 19.

\section{Modi.}

$\$$ 39. Konjunktiv. Der blofse Konjunktiv findet sich noch in Wunschsätzen und vertritt hier die 3. Person des Imperativs.

Beispiele: mire ke el azniko es suion I, 9; ę̣os tęngan bį̣n II, 33.

In kale sẹa ke no es el Diq . . III, 27 drückt er eine unsichere Behauptung aus.

§ 40. Gerundium. Das Gerundium wird jsp. sehr häufig gebraucht. Oft steht es in Verbindung mit $\boldsymbol{e n}$.

Wie asp. ist das Subjekt oft aus dem Zusammenhang zu ergänzen (vgl. Zauner § 187 ).

Beispiel: en ğugando [las kriaturas] I, 3.

In î kręo ke en i̇ebándola b'a tener un buęn regalo II, 29 vertritt das Gerundium (wie auch oft frz.) die Protasis eines Bedingungssatzes. 


\section{B. Nomen.}

§ 41. Artikel. Vor $D_{i g}$ findet sich regelmärsig der bestimmte Artikel (vgl. auch Subak, Judensp. S. 130), während ihn weder das Asp. noch das Hebräische in diesem Falle zu setzen pflegt.

Beispiel: el Dìg ke se tęge III, 133.

Kas us.

§ 42. Dativ. Der Dativ oder vielmehr die ihn ersetzende Wortgruppe " $a+$ Substantiv" steht bei Personalobjekt bereits häufig für den Akkusativ.

Beispiele: iamar a las tres ermanas III, 23; iamar a la ermana grande III, 40; tomg al fižo III, 43. Doch findet sich auch noch der Akkusativ: salbar lqdas estas personas III, 175; estás tįendo ... una niña III, 175; bido su ermana III, 186; fizo eskondęr los dgz ermanikos III, 199; bid̈o su fiža III, 190.

\section{Pronomen.}

§ 43. Personalpronomen. I. Wie im Asp. tritt bei Personalobjekt noch nicht das Dativpronomen für den Akkusativ ein.

Beispiele: las embip a iamar III, 24; loz bido III, 136; lo bido III, 163 ; las menasp III, 28; la kería III, 53.

2. In der höflichen Anrede wird die 3. Sing. im Sinne der 2. Sing. gebraucht.

Beispiel: mire ke el azniko es suizo I, 9.

3. Auffällig ist die Doppelsetzung des Objektspronomens in: los puedo dispareserlos III, 147 und la fug a epkontrarla III, 161 .

4. Der ,ethische Dativ' findet sich wie asp. (Hanssen $\S 47,10$ ): te to bas a kregr III, 121.

8 44. Possessivpronomen. In der 3. Person steht der Plaral des Possessivpronomens anch, um eine Mehrheit der Besitzer bei singularischem Besitz zu bezeichnen: sus padre III, 206; suz madre III, 202.

Mit Recht ist tk. بابالرى babalary zum Vergleich herangezogen worden (Wagner § 93, Lamouche S. 984).

§ 45. Relativpronomen. ke steht auch als relatives Adverb und bezeichnet a) die Zejt [Hanssen $\$ 54,11$ ]: un dia ke III, 106; de la mar̃ana ke III, 166; en kada tẹs ke II, 12.

b) den Ort: este lugar ke se fun la niña III, 156. 
ChaRAKTERISTIK DES JUDENSPAN. DIALEKTS VON SOIONIKI. 685

\section{Dritter Abschnitt.}

Syntax.

\section{Kapitel I: Hilfsverben.}

§ 46. I. Perfekt und Plusquamperfekt werden wie port. mit dem Hilfsverb tenęr anstatt mit aber gebildet, z. B. no la tęngo nunka sentido kantar (abia prometido III, i 30 Gallizismus). Doch begegnet überhaupt die Umschreibung in der gesprochenen Sprache nicht häufig. Unsere Texte ziehen einen ausgedehnteren Gebrauch des Praeteritums und des Imperfektums vor (vgl. \$§ 36-37).

2. Dagegen ist die Umschreibung des Futurs und Konditionells durch $t q$ bezw. $i a+i n f$. sehr häufig und scheint auf dem Wege zu sein, diese Tempora allmählich vollständig zu verdrängen.

Ebenso steht es mit der Umschreibung des Praesens durch nestar + Gerundium ${ }^{*}$, der auch Subak (Judensp. S. I 30) eine solche Zukunft prophezeit.

\section{Kapitel II: Negation.}

§ 47. Wie im Asp. steht ein nq bei ninguno, auch wenn dieses dem Verbum vorangeht (vgl. Zauner $\$ 93$, Hanssen 860,3 ).

Beispiele: ke ningunos ${ }^{1}$ ne asįgndan lus III, 2 ; ke ningunos 1 ne mॄz tan a tęr III, 8.

\section{Kapitel III: Wortstellung.}

$\S 48$. Stellung des Objekts. Stark betontes Objekt steht vor dem Verbum und erscheint selist am Satzanfang.

Beispiele: ke las kriaturas lq ke sigenten ambęzan II, 2; ke esta karapusa no es el Dì ke me la'mbịq II, 27; ke a las kriaturas kale ambezadio II, 32 ; tod̆o ẹsto el fizo del ręi estata sintięndo III, 22.

\$ 49. Stellung des Objeitspronomens. 1. Enklisis des Objektspronomens ist die Regel:

a) beim Imperativ: estáte kaiada $\mathrm{III}, 65$; estáte en repqzo III, 147; bále III, 153; aigrnate III, $164 ;$ akatuabate III, 172; fuįqudobos I, 8; kitándolo I, 10; asperándobos III, 204 ;

b) beim Infinitiv oder Gerundium: para labarse III, 180; para abrasarlo III, 187; por teggarse III, 45; por engrasiarlo III, 193; de aserkarle III, 172 (aber: a lugar de le dar la kriatura III, 46); en tięndolas III, 129; en ietandola II, 29.

2. In den Gruppen nir + inf." und nestar + Gerundium" schliefst sich das Objektspronomen immer an ir und estar an.

I Auffälig der Plural von ninguno, wohl nach algunos. 
Beispiele: la to a aferar II, II; mpz tamos a arodear III, 7; ne moz tan a ber III, 8; is le ia a fazer a ęl III, 15; io le ia a parir III, 20; te ia a fazer III, 29, 31; te ta' 'car de kasa III, 66 usw. se estatan abruensando III, 28; de estarlos admirando III, 98; te este diziżendo III, 109; las este entendięndo III, 132 ; la estuto asperando IIl, 160.

3. In den Verbindungen des Infinitivs mit andern Verben steht der Gebrauch teils nicht fest (wie z. B. bei pyeder), teils erlaubt die geringe Anzahl der vorhandenen Beispiele nicht, Regeln hierüber aufzustellen.

benir: la binieron a buskar III, $17 \mathrm{I}$.

detgr: ke detęmos de fazerle III, 2 IO.

embiar: las embiq a iamar III, 24; la embige a iamar III, 59,77 .

empesar: empesaron a enčirse III, 173.

kerer: no te kigro tęr mal III, 109; me kiegres fazer un plazeriko Ill, 149.

pueder: se puede kitarlo I, 6; puedes fazer deztacearlo a el rei III, IO3; si se lo puedes kitar III, 170; los puedo dispareserlos III, 147 (vgl. $\$ 43,3$ ).

ser: la fus a egkontrarla III, 16 I (vgl. $\$+3,3$ ).

tenęr: no tenẹnos k'a demandarle III, 208 (vgl. Anm. zur Stelle).

$\S 50$. Stellung der Negation. ne te kig̨ro ter mal III, 109 zeigt die Negation beim Verbum finitum, während es logisch zum Infinitiv zu stellen wäre (vgl. Tobler, V. B. I, S. $196 \mathrm{ff}$.)

\section{Glossar.}

\section{A}

aboltar 'wenden' III, 186. atryensarse 'sich schämen' III, 28. Die starke Kontraktion durch den Affekt verständlich, mit dem 'ss torguensa', oder mit Metathese 'Treguchsa' und kontrahiert 'Zrųnsa' gespro- chen zu werden pllegt. ačetar 'annehmen' III, 59 ital. agera 'jetzt' III, 132 asp. Vgl. Wagner \& I I 2.

ağidearse 'sich erbarmen' III 86

tk. آجيين adjymaq Tewfik 2, III.

Vgl. § 33 .

akatidar 'verbieten' II, 5 akatidarse 'sich in Acht nehmen' III, I 72 . M.-L. Wb.
1793 erwähnt aport. cavidarse sich in Acht nehmen' neben, neuport. cavidar 'entgegentreten'. Vgl. \& 33.

amd 'aber' III, 185 tk. Lil amma Tewak 37, I.

ambezar 'lehren' II, 32; III, I 5 I

- 'lernen' II, 3. Vgl. \& 3, I.

- Fehlt bei M.-L. Wb. 4536,2 [invitiare].

$a_{n}=e n$ ? Vgl. Anm. zu III, 18. Bedeutung wie ' $a$ ' III, 18,20 , 24, 209.

ansina 'so' III, 38, 77, 88 usw. Vgl. \$ I 4 , 1 .

apẹnas ke 'kaum' III, 182 ; asp.

Vgl. Hanssen § 62, 12. 
aprometir 'versprechen' III, 68.

Vgl. § 33 .

aretar 'anhalten' III, 149 frz. aritar 'ankommen' III, 162 asp. llegar salon. unbekannt.

arodearse 'sich herumstellen, herumsetzen' III, 7. In dieser Bedeutung auch asp.?

asendęr 'anzünden' III, 2,24 asp. - nsp. encender. Vgl. Wagner $\S 134$.

asperar 'erwarten' III, 155 u. 160. - asperarse 'warten' III, $204=$ esperar. Vgl. § 3, I. atornar 'zurückkehren' 1,5 . atornarse 'zurückkehren' III, 164. Vgl. § 33 .

atrebirse 'wagen' III, 179= atreberse. Konjugationswechsel?

\section{B}

bito 'lebhaft' III, 6, 62. Vgl. $\S 5 \mathrm{~d}$.

bolado de la gustada 'voller Freude' II, 18. Vgl. Anm. dazu.

burako 'Loch' III, II port.? Vgl. Wagner \& 135 .

buškar 'suchen' III, 171 . Vgl. $\S 12$.

B

tẹs, una bęs ke 'da ja' III, Ig6. tesir 'Wesir' II, I - nsp. visir. salon. wie tk. rj, zesir Tewfik 363, III. Konstant. bizir erk]ärt sich nach $\$ 3,2$.

$\check{\mathbf{C}}$

¿̌č̌ ẹ́k 'Blume, Blüte' III, 96. Tr. خيجن Cicek Tewfik 124, I. cobán 'Hirt' III, 83, 85. Pers.tk. ioban Tewfik I22, I.

\section{D}

delantre 'vor' II, 5, 9; III, II 3 usw. = delante. Vgl. \& I4, 3 . demandar 'fragen' II, 21,30 ; III, 27, 56, 190, 209. - Die
Konstruktion mit dem Dativ wohl Gallizismus.

dertis 'Derwisch' III, 163, 168. - Pers. - tk. ذرويش derwis Tewfik 149, III 'mohammedanischer Mönch, Derwisch'.

deztačear 'abbringen von' III, 103. Nach Mitteilung von $\mathrm{Hrn}$.

Dr. Wagner zu tk. waz geimek Tewfik 360, II.

dezmazalađ̃o 'unglücklich' III, 80 - bei Cervantes belegt. Vgl. Wagner $\$ 176$ und dies Glossar unter mazal.

\section{$\mathbf{E}$}

ečarse 'sich zu Bett legen' III, I6, 30; oft für ečarse a la kama.

ešpionar 'ausspionieren' III, 9. Gallizismus für espiar.

\section{$\mathbf{F}$}

fičisera 'Hexe' III, 102, 104 usw.; nsp. hechicera. Vgl. §3, 2. fezezika 'kleiner Fes, Feslein' II, IO; Diminutiv von la fes II, 12 (in Konstantinopel $f e x$ ) $=$ tk. wes Tewfik 247, I. Vgl. § 32 .

fin a 'bis' III, 191. Aus dem Ital.?

G

gazeziko 'Petroleum-Lämpchen' III, 7. Auf dem ganzen Balkan bedeutet gas 'Petroleum'. Züm Diminutiv s. $\$ 32$.

Ğ

ğudįg 'Jude' II, 8, r6. Vgl. § 1 .

H

hahán 'Rabbiner' I, 1, 5 usw.; hebr. 13 , wovon ik. pli haham Tewfik 1 35, III. Der dem Spanischen ungewohnte Auslaut ist in den nächstverwandten Konsonanten übergegangen, wie in šalọn I, 7. Vgl. auch 
den Wandel quem $>$ quien, cum $>$ con. In Üsküb heirst es haham. hanúm, auch hanú, 'Fräulein'

III, I 5O; tk. خانم chanym Tewfik 137, II 'Frau'. 'Fräulein' tk. eigentlich غانم chanym kyz

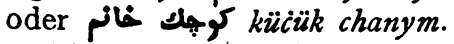
harači 'HuL' II, i6. Türkischen

Ursprungs nach Danon (Keleti Szemle I 904, S. I I I), der haracin notiert und es mit 'bonnet de lingerie' übersetzt.

\section{I}

itar 'auf die Weide führen' III, I13. Wohl Nebenfurm von ietar über *iitbar (dieses nach \& 3,2 ).

inda 'noch' III, 16,18 usw. -

Nach Hanssen $\S 58,6$ begegnet inda abgesehen vom Port. auch im Neuasturischen.

in ' 'trotzdem' III, 179, 185 ; tk. 8lin jine. Tewfik 383, I'wieder, ebenfalls, dennoch' Zenker 969, III 'nichtsdestoweniger'. Salon. auch ing ke für aunque, wie ing kon todo neben aun kon teqdo.

jertado 'Rasen' III, I12, 114 usw. - nsp. hierbasal. M.-L. Wb. 4109 erwähnt asp. herbadgo 'Weide'.

\section{$\mathbf{K}$}

kal 'Synagoge' 1, 2, 5. Hebr. לדৃ? kahal.

kale 'es ist nötig, man mufs' II, 27, 32 usw. asp.

karapusa 'Hut' II, 16, 17 usw.

Asp. carapuza, nsp. caperuza.

Vgl. Wagner \$ 134 .

keręr 'wolien' mit $a+$ inf. III, 36 . kolái 'leicht' III, 10+, 208; tk. qolaj Tewfik 269, I.

konák 'Palast' II, 8 ; tk. قوناق gonaq Tewfik 270, II. kontunear 'fortfahren' III, 117 . An Stelle von continuar mit Metathese ( $\$ 17)$ und Suffixwechsel.

kriadera 'Amme' II, 5, 6 usw. Auch von Subak (Salon. $\$ 32$ S. 10) notiert.

kriatura 'kleines Kind' II, 2, 20 usw. Steht 2 war nicht wie nsp. nur im Sinne von 'neugeborenes Kind', bezeichnet aber immerhin ein 'kleineres Kind'. Allgemeiner fizos. niño bedeutet im Sing. und Plur. nur 'Knabe'.

kuбižar 'bedecken' III, 18, 32. Nsp. cobijar. Vgl. § 3, 2.

L

labo rađẹro 'fleifsig' III, 3; asp.? ladto 'Seite' de lado III, I06; a lado III, 97.

\section{M}

ma 'aber' III, 25 usw. Ital. ma auf dem Umwege über türk. und griech. Kaufleute ins Jsp. gedrungen (Wagner $\S 138$ ).

mazál 'Geschick, Bestimmung' II, II; III, 74; nhebr. לৃֶ mazal. Vgl. Wagner $\$ 176$. Davon desmazalado und üskübisch masalqzo.

men as ar 'bedrohen' IU, 28; asp.nsp. amenasar.

meqijo 'Stirn' III, 21, 35 usw. Auch 'Gehirn' (man verlangt beim Schlächter luęnga $i$ mepio) und 'Gedächtnis' (kign no tìgne megio ke tenga pačás ['Beine', tk. 8lọ paica 'Tewfik 78, II]). moab ęt 'Unterhaltung' II, $\mathbf{x}$; tk.arab. محتبّ muhabbet. Tewfik 308, II. Vgl. § 5 a, $\gamma$.

mu६to 'neu' III, $7 \mathrm{I}=$ nueto § I $5,2$. 
$\mathbf{N}$

$n \varphi$ an Stelle von asp. non ist bereits die herrschende Form, doch begegnet auch noch nqn.

\section{0}

odá 'Zimmer' III, 70, 200; tk. 8b,l oda Tewfik 47, III. Man hört auch $u d d$, dieses nach $\$ 3,2$.

\section{$\mathbf{P}$}

pará, Plur. parás 'Geld' III, 73; tk. 8, para Tewfik 79, I. pardonar 'verzeihen' III, 53 mit Akkusativ konstruiert. Statt perdonar, wohl Gallizismus. pensa đa a'Gedanke' III, 2 I 5 ; asp.? por 'für, um ... zu' mit finalem Infinitiv III, 45 u. 193. pranso 'Mittagessen' III, 199 ital. pregen, ečar un pregen 'ausrufen lassen' II, 24; III, I; asp. pręsto 'schnell' I, 10; asp.

pųqpo 'Leib, Körper' III, 2 I 3 für cuerpo. Vgl. \$ 16.

punto 'Augenblick' II, 23; III, 147 usw. Vgl. lat. punctum temporis.

S

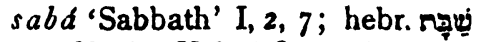
sabbath. Vgl. \& 8 u. § $5 \mathrm{a}, \gamma$.
Der Anlaut wohl durch sábado $<$ lat.-griech. sabbatu mbeeinfluist.

sestiko 'Körbchen' III, 1 53; nsp. cestico.

siklear se. 'sich langweilen' III, I3; 'sich beunruhigen' III, 146; tk.

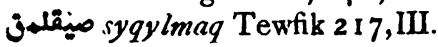

Vgl. \$ 33.

\section{演}

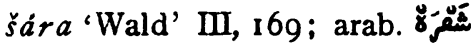
šara $=$ nsp. jara, Vgl. M.-L. Wb. 7674 und M. L. Wagner ZRPh. XL, $547 \mathrm{f}$.

\section{$T$}

topar 'finden' II, 24; hallar salon. nicht vorhanden.

tosego 'Gift' III, 108; nsp. tosigo. traį̣r 'führen, treiben' III, I15 $=$ traer. trajer auch westleon. Vgl. Hanssen S. 82.

\section{U}

uqrko 'listig, verschlagen' III, 6 (el kerko = 'Teufel'). Das Adjektiv bedeutet nicht, wie asp., 'traurig'.

una, on una kon 'zusammen' III, 188.

Walter Simon. 